\title{
A stochastic study of the collective effect of random distributions of dislocations
}

\author{
B. Gurrutxaga-Lerma \\ Trinity College Cambridge, Cambridge CB2 1TQ, UK and \\ Department of Engineering, University of Cambridge, \\ Trumpington St, Cambridge CB2 1PZ, UK
}

\begin{abstract}
The effect that random populations of dislocations have on a material is examined through stochastic integration of a random cloud of dislocations lying at some distance away from a material point. The problem is studied in one, two, and three dimensions. In 1D, the cloud consists of individual edge dislocations placed along the real line; in 2D, of edge dislocations and edge dipoles on the plane; in $3 \mathrm{D}$, of dislocation loops. In all cases, the dislocation cloud is randomly distributed in space, associated to which several relevant physical parameters, including the material's slip geometry, the dislocation's sign, and its relative orientation, are also stochastically treated. A fully disordered population, i.e., one where the dislocation's signatures and orientations are entirely random, is first studied. It is shown that such disordered systems entail a strong indeterminacy in the collective stress fields, which here is solved by enforcing mass conservation locally. In $2 \mathrm{D}$, this is achieved by modelling a cloud of edge dipoles instead of individual dislocations; in 3D, this is naturally guaranteed by the modelling of closed dislocation loops. The long-range fields of the dipoles in 2D and of the loops in 3D is modelled via their multipolar force expansions, which greatly simplifies the analytical treatment of the problem. The cloud's effect is then studied by performing the stochastic integration of the multipolar fields via Campbell's theorem. The local order, but not the magnitude of the dislocation density, is shown to be critical in contributing to the plastic relaxation of the material: fully disordered systems are shown to self-attenuate, leading to plastic neutrality; ordered and partially ordered systems, achieved when dislocation signatures are aligned, display a direct relationship between the dislocation density and the average stress shielding the material. We establish and generalise the conditions that a system of dislocations must fulfil to display Taylor's equation and the Hall-Petch relation, and offer adequate scaling laws related to this.
\end{abstract}

\section{INTRODUCTION}

This article is concerned with the study of random populations of dislocations, and their long range effects on any crystalline material that may exhibit such arrangements, or the dislocation microstructure of which may be modelled as being random. The idea that a population of dislocations may be loosely regarded as randomly distributed throughout a crystalline sample is relatively commonplace[1-3], and long rooted in the experimental observation of dislocation microstructures $[4,5]$. Such arrangements have often been cited as being crucial in the study of dislocation motion against obstacles [6-8], and underpin such theories as the distribution of Frank-Read sources in a crystal [9-11] or the internal ordering of the forest of dislocations, which experimental observation [12, 13], theoretical studies [14, 15], and modern simulations $[16,17]$ typically assume to be entirely random. Furthermore, the statistical study of collective of dislocations has received attention in the past, usually aimed at developing continuum theories of plastic flow by statistically homogenising the slip carried over by the dislocations[18-22]; usually. in these cases the dislocation densities are not treated explicitly, but its spatial evolution is studied through varied physical and statistical considerations[23-25]. Finally, the assumption that a random distribution of dislocations may be employed as the starting point to study salient features of its evolution under varied geometrical and loading conditions is pervasive in modern simulations of discrete dislocation dynamics [26-34].

One may therefore wonder whether in considering such random arrangements of dislocations, one is in fact implicitly assuming a specific internal residual stress state in the material or, vice versa, whether such random distributions of dislocations may be constructed so as to respond to or in such a way as to induce a specific stress state in the material. The greatest difficulty in this regard appears to be the averaging of the collective, long-range fields of the population of dislocations [3]. This is because the population of dislocations may be entirely random, but their inherent elastic fields are slowly decaying, and will be affected by the random position of each dislocation line. Previously, Kröner [35] had already suggested that statistical descriptions of the collective behaviour of dislocations ought to be described in terms of the probability to find a dislocation in a specific position relative to another dislocation. This idea led to

\footnotetext{
*bg374@cam.ac.uk
} 
the development of a statistical description of dislocation dynamics based on pair correlation functions, as first done by Groma [36] in the context of X-ray line broadening due to disordered distributions of dislocations and, subsequently, by Groma and Bakó[37] with stochastic ensembles of parallel edge dislocations. Further work by El Azab [18], Zaiser et al. [38], Kratochvíl and Sedlácek [19] amongst many others, have focused on computing the pair correlation functions describing the dislocation density distribution and the general probability distribution functions, aiming at describing plasticity evolution laws (see [39-41]), and the energetics of dislocation ensembles [42-44]. Recently, Berdichevsky [45] has offered an expression for the regularised energy of a random set of dislocation lines, Zaiser[44] used an elastic energy functional approximation to study two and three dimensional ensembles of dislocations.

In the statistical approach developed by Groma and coworkers[37], the stress state is obtained from the probability distribution of the stress field, which in turn is dependent on the conditional probability to find dislocations at a given location, and is therefore not available in a general case [46], but may be obtained from X-ray diffraction data, dislocation dynamics simulations [39, 40], or for specific kinds of dislocation ensembles[41, 47]. For instance, Csikor and Groma [47] computed it for disperse arrangements of uncorrelated dislocation dipoles. Another possible way of evaluating the stress state would be to perform a set of Monte Carlo style simulations where the collective average fields of a vast number of random realisations of a stochastic collective of dislocations are computed, such as those obtained via fibre processes by El Azab et al.[48]. In that case, one would produce a random population of dislocations, comprising their spatial positions, crystallographic orientations, signs, loop shapes, etc, and evaluate that realisation's collective stress field on a point. This would entail the computation of the individual stress field of each dislocation in the realisation, and in order to get statistically significant averages, the compounded average of many such simulations would need to be provided. The latter would offer a measure of the average 'macroscopic' stress field, and of its variance. These would depend on the way the dislocations and their inherent properties are distributed in space, so by modifying the latter different kinds of average stress fields could be computed.

Arguably, any such calculation would have vast computational cost, particularly in three dimensions, and so long as a few underlying assumptions with regards to the nature of the spatial distribution of dislocations and to their long range fields are introduced, this article intends to show that they would be entirely unnecessary: as we shall argue here, it is possible, through stochastic integration, to evaluate analytically many of the properties, including the average stress fields, induced by a random population of dislocations.

In particular, in this article we are interested in evaluating the long range effect of any such random distribution of dislocations, and in determining whether they contribute to the global plastic relaxation of the material and, if so, how. In computing the average stress field of a random collective of dislocations, we are computing the amount of plastic relaxation they contribute to. Hence, the yield point and the subsequent plastic hardening will be directly proportional to this collective stress field.

In this work, we shall focus on dislocation clusters in the bulk, and as such deal with bulk plastic response; plasticity aspects pertaining finite systems and finite size effects are not considered here. We propose a stochastic method based not on the general computation of pair correlation functions and probability functions, but on the theory of spatial point processes to evaluate the collective effects of such randomised arrangements of dislocations on the macroscopic average stress. We are not concerned with how the random dislocation microstructure came to be, but on how, given a few stochastic assumptions (in particular, that the dislocations be distributed as a spatial point process), any one given population of dislocations may influence the salient features of their collective stress field. We therefore merely assume that such distribution of dislocations exists, that its spatial arrangement may be modelled in some particular way (say, localised in 'walls' of dislocations, or entirely disperse throughout the sample), and we evaluate its collective effect through stochastic integration of the individual stress fields of each dislocation in the sample. However, this does not prejudice comparison between the populations of dislocations under consideration and those often mentioned in experiments and simulations to be random, such as for instance those recently reported by Gunkelman et al.[49], given that many of the features of a deterministic population of dislocations may be approximated through appropriate choice of the statistic distribution of the parameters defining the random cloud of dislocations.

The article is structured as follows. In section 2, we discuss the case of a one dimensional array of dislocations, and highlight the differences between assuming particular orders leading to Taylor's equation, and perfectly random arrangements of dislocations. In section 3, we extend this analysis to the planar case, where we consider disordered populations of straight edge dislocations; we show that unless mass conservation is strictly enforced locally, we run into statistical indeterminacies which we are able to solve by introducing the multipolar expansion of a (random) dipole of dislocations. We then go on to study the collective effect of such cloud of dislocation dipoles under various assumptions, showing that Taylor style equation, i.e., that the yield point be directly proportional to the square root of the dislocation density, cannot be recovered unless particular spatial arrangements of dislocations are considered; such 
arrangements will result in appropriate scaling laws related to the internal average parameters of the random cloud of dislocations. Subsequently, in section 4 we extend the study to three dimensions. We model the long range fields of random dislocation loops via their multipolar field expansion, and again estimate their collective effect on a given point. As before, we are able to establish the circumstances in which Taylor's equation is recovered, leading to an additional number of scaling laws for the dislocation microstructure. Finally, in section 5 we discuss the significance of this work's findings, offering some remarks alongside our conclusions.

\section{PLASTIC RELAXATION INDUCED BY RANDOM CLOUDS OF DISLOCATIONS IN ONE DIMENSION}

Given that the stress fields of dislocations decay with $1 / r$ (i.e., the inverse of distance) [8], their effect in relaxing a given material point can arguably be considerable. At the same time, the superposition of the long range field of two dislocations of equal magnitude but opposite sign may on a first approach appear to cancel their respective effects, and not contribute to the plastic relaxation of the material. In fact, in the latter case a contribution would remain: the dipolar field expansion of such dipole of dislocations will decay with $1 / r^{2}$, and be directly proportional to the separation $l$ between the two dislocations forming the dipole (see section 3 and [50]); this means that the two dislocations in a dipole do not fully shield one other in the long range.

Now imagine that the population of dislocations is large, and that mass is conserved (i.e., that the number of dislocations of either sign is the same). If the long range effect of individual dislocations and of dislocation dipoles (if mass is conserved it is possible to group all dislocations into dipoles) remains, it becomes increasingly difficult to judge whether the population of dislocations will promote plastic relaxation, plastic shielding, or whether it will self-attenuate and remain plastically neutral. Here we shall study with increasing level of randomisation how either of this cases may arise in a one dimensional array of dislocations.

\subsection{The $1 \mathrm{D}$ case}

Assume that a material point of interest, is located at $y=0$, and that for $x>0$ there lies a population of straight edge dislocations. The first dislocation is placed at a distance $R$ from the origin, and thereafter the distance between successive dislocations is given by $r_{n}$. The only stress field component acting at the origin is $\sigma_{x y} \equiv \tau$, which is given by [8]

$$
\tau=D\left[\frac{s_{0}}{R}+\frac{s_{1}}{R+r_{1}}+\frac{s_{2}}{R+r_{2}}+\ldots\right]=D \sum_{n=0}^{\infty} \frac{s_{n}}{R+r_{n}}
$$

where $D=\frac{\mu B}{2 \pi(1-\nu)}$, for $B$ the magnitude of the Burgers vector, $\mu$ the shear modulus and $\nu$ Poisson's coefficient.

In the most general case of interest here, $s_{n}$ and $r_{n}$ are both random variables and, consequently, so is $\tau$. In particular, so as to guarantee mass conservation, we must impose that $s_{n}=\{+1,-1\}$ with equal probability $P_{s_{n}}=1 / 2$ (i.e., following Rademacher's distribution), whereas $r_{n}$ is allocated by some probability distribution, with the sole conditions that it be bounded between $(0, \infty)$.

Prior to that, however, it might be illuminating to study the case in which $s_{n}=(-1)^{n}$ (i.e., the sign of the dislocations is alternating), and where $r_{n}=n \cdot L$, for $L$ some fixed spacing between the dislocations. This is in fact a one dimensional version of the situation originally considered by G.I. Taylor in his seminal work on dislocations [51]. In that case, the sum can be evaluated exactly [52]:

$$
\tau=\frac{D}{L} \Phi\left(-1,1, \frac{R}{L}\right)
$$

where $\Phi(-1,1, R / L)$ is a Lerch transcendent[53], which means that (to first order) $\tau$ decays with $1 / L$, thereby confirming Taylor's equation that $\tau \propto \sqrt{\rho_{\text {dis }}}$ so long as $L \propto 1 / \sqrt{\rho_{\text {dis }}}[51,54]$.

As we shall see in the following, disordering the population of dislocations radically alters this classical result. To begin with, we may assume that the dislocations remain evenly spaced by some deterministic $r_{n}=L$, but that their sign is now a Rademacher distributed random variable. In that case, the mean stress at the origin is simply zero:

$$
\bar{\tau}=E[\tau]=\sum_{i=0}^{\infty} \tau_{i} \cdot P\left(\tau_{i}\right), \quad \text { where } \quad \tau_{i}=\left[D \sum_{n=0}^{\infty} \frac{s_{n}}{R+n \cdot L}\right]_{i}
$$


Since $n \in \mathbb{N}, P\left(\tau_{i}\right)=0 \forall i \in \mathbb{N}$, then $\bar{\tau}=0$. The variance in this case is (see [52]):

$\operatorname{Var}[\tau]=E\left[\tau^{2}\right]-E[\tau]^{2}=D^{2} \sum_{n=0}^{\infty} \frac{s_{n}^{2}}{(R+n \cdot L)^{2}}=D^{2} \sum_{n=0}^{\infty} \frac{1}{(R+n \cdot L)^{2}}=\frac{D^{2}}{R^{2}} \sum_{n=0}^{\infty} \frac{1}{(1+n \cdot d)^{2}}=\frac{D^{2}}{L^{2}} \psi^{(1)}\left(\frac{1}{d}\right)$

where $d=L / R$, and $\psi^{(1)}$ is the first derivative of the digamma function, i.e., $\psi^{(1)}(x)=[\ln \Gamma(x)]^{\prime}$. Because the series is absolutely convergent, the variance is bound to be finite. The case when the first dislocation is far away from the crack tip, i.e., when $R \gg r$ (and $d \ll 1$ ), enables a direct asymptotic limit to the variance, whereupon the standard deviation is found to be of order

$$
\sigma_{\tau}=\frac{D}{L}(1+\text { h.o.t. })
$$

that is, for a point sufficiently away from the dislocation cloud, the standard deviation on the stress experienced by the point is dominated by the spacing between dislocations. Still, we observe that such disordered population of dislocations has no net effect on the origin, unlike in the fully ordered 'lattice' Taylor model.

In the event the distance between dislocations, $r_{n}$, is a random variable as well, the resulting process is in effect a marked point process, thereby enabling us to exploit the Campbell theorem (see [55]) as follows. We define the collective random variable $\boldsymbol{x}=(r, s) \in \mathbb{R} \times \mathbb{M}$, where $\mathbb{M}$ is the mark space added to each $r_{i} \in \mathbb{R}$. In this case, $\mathbb{M}=\{-1,1\} \subset \mathbb{Z}$ is the mark. The point process $N$ in $\mathbb{R} \times \mathbb{S}$ is defined as $N=\left(r_{0}, s_{0}\right),\left(r_{1}, s_{1}\right), \ldots \equiv \boldsymbol{x}_{1}, \boldsymbol{x}_{2}, \ldots$, and such that we are interested in the random variable

$$
\tau=\frac{D}{R} \sum_{\boldsymbol{x} \in N} \frac{s}{1+r / R}=\frac{D}{R} \sum_{\boldsymbol{x} \in N} f(\boldsymbol{x}), \quad \text { with } \quad f\left(\boldsymbol{x}_{i}\right)=\frac{s_{i}}{1+r_{i} / R}
$$

Then, by virtue of Campbell's theorem,

$$
\bar{\tau}=E[\tau]=E\left[\frac{D}{R} \sum_{\boldsymbol{x} \in N} f(\boldsymbol{x})\right]=\int_{\mathbb{R} \times \mathbb{S}} f(r, s) \Lambda(\mathrm{d} r, \mathrm{~d} s)
$$

where $\Lambda(\mathbb{R} \times \mathbb{S})$ is the intensity measure for the marked process, about which so far we have assumed nothing further. Here the mark space is $\{-1,1\}$ with equal probability for either of the two signs, so the mark $s_{n}$ follows Rademacher's distribution:

$$
\varsigma(s)=\frac{1}{2}[\delta(s+1)+\delta(s-1)]
$$

the expectation of which is $E[\varsigma(s)]=0$ by construction. Since $r$ and $s$ are independent from one another, the product measure factorises, whereby

$$
\bar{\tau}=\int_{\mathbb{R}} \Lambda_{r}(\mathrm{~d} r) \int_{\mathbb{S}} f(r, s) \varsigma(s) \mathrm{d} s=0
$$

irrespective of the spatial distribution of the underlying point process. $^{1}$

The variance is not available for a general point process, but may be estimated for a number of cases, depending on the assumptions we are ready to make about the distribution of points $r$. We may find a general upper bound to it by invoking the Cauchy-Schwarz inequality, followed by the Campbell theorem, as follows

$$
\begin{aligned}
\operatorname{Var}[\tau] & =\left(\frac{D}{R}\right)^{2} \int_{\mathbb{R}^{2}}\left(\sum_{\boldsymbol{x} \in N} f(\boldsymbol{x})\right)^{2}\left[\frac{1}{2} \delta(s+1)+\frac{1}{2} \delta(s-1)\right] p(r) \mathrm{d} \boldsymbol{x} \\
& \leq\left(\frac{D}{R}\right)^{2} \int_{\mathbb{R}^{2}} \sum_{\boldsymbol{x} \in N} f^{2}(\boldsymbol{x})\left[\frac{1}{2} \delta(s+1)+\frac{1}{2} \delta(s-1)\right] p(r) \mathrm{d} \boldsymbol{x}=\left(\frac{D}{R}\right)^{2} \int_{\mathbb{R}_{r}} \mathrm{~d} r \frac{p(r)}{(1+r / R)^{2}}
\end{aligned}
$$

If $R \gg r$, then we may asymptotically expand the integral in the upper bound about $r / R=0$, which renders

$$
\operatorname{Var}[\tau] \leq\left(\frac{D}{R}\right)^{2}\left(1-2 \frac{\bar{\Lambda}}{R}+\text { h.o.t. }\right)
$$

\footnotetext{
${ }^{1}$ We also note that in the event the dislocation mass is not conserved, i.e., if the probability of positive and negative dislocations is not exactly the same, $\bar{\tau}=\infty$, irrespective of the point process
} 
where $\bar{\Lambda}$ is the average intensity of the point process. If we assume that the spatial point process' intensity follows a uniform Poisson distribution of density $\lambda$, then $\operatorname{Var}[\tau] \leq\left(\frac{D}{R}\right)^{2}\left(1-2 \frac{\lambda}{R}+\right.$ h.o.t. $)$, where $\lambda$ in this case is the average counting measure, i.e., the dislocation density.

We therefore conclude that in the one dimensional case, the effective plastic relaxation on a given fixed point due to the random cloud of dislocations will on average be zero, and the variance of this shielding stress will be bound by the average distance between dislocations in the cloud. In addition, we may recover Taylor's equation, but in order to do so we must impose a strict spatial and sign order in the population of dislocations. Otherwise, a randomised pattern of dislocations does not appear to follow Taylor's equation in one dimensions. Hereafter, we shall refer to the situation where the average stress field of the cloud of dislocations is zero as 'plastic neutrality'.

\section{PLASTIC RELAXATION INDUCED BY RANDOM CLOUDS OF DISLOCATIONS IN TWO DIMENSIONS}

Clearly, the 1D case is highly degenerate: one does not expect dislocations to be aligned solely in such a specific direction as the axis of abscissae. Still, it serves as an illustration of how the collective effect of a random cloud of pre-existing dislocations over a given point may be statistically characterised. In particular, we have shown that the case of random populations of dislocations may be treated as marked point process, and that the mean stress field evaluated by means of Campbell's theorem.

In the following, we shall extend the one dimensional study of random collectives of dislocations to two dimensions. We shall focus on clusters of dislocations in the bulk, i.e., in the absence of a free surface, and highlight a number of conditions that need to be enforced to obtain meaningful statistical averages of the stress fields. The radically opposite case to this one, i.e., one with a fully ordered system of dislocations in 2D forming a lattice of dipoles along a single specific direction of slip, is the classical study by Taylor [51], which as in the $1 \mathrm{D}$ case leads to Taylor's equation that $\tau \propto \sqrt{\rho_{\mathrm{dis}}}$; as we shall see, disordered systems do not necessarily exhibit such property, unless a specific number of conditions are met.

\subsubsection{Fully disordered system}

The most straightforward generalisation of the 1D case to two dimensions would be to allow a random cloud of straight edge dislocations to surround a given point of interest, their sign and positions being assigned independently and at random, without further conditions. Here, we employ polar coordinates for convenience. In that case, we place the point of interest at the origin $(r, \phi)=(0,0)$, and locate any given dislocation $i$ at some radial distance $r=R+r_{i}$, where $R$ is the minimum distance between any dislocation and the origin (and $r_{i} \in \mathbb{R}^{+}$). The angle $\phi_{n}$ describes the angular location of the dislocation line relative to the origin's. The dislocation is oriented relative to the this plane by an angle $\theta_{n}$, which specifies the orientation of the dislocation's slip plane.

Then, relative to the origin, the stress field on the origin due to such a cloud of dislocations $n=0,1, \ldots$ is given by

$$
\begin{aligned}
\sigma_{r r} & =-D \sum_{n=0}^{\infty} s_{n} \frac{\sin \left(\phi_{n}-\theta_{n}\right)+\cos \left(\phi_{n}-\theta_{n}\right) \sin \left(2 \phi_{n}\right)}{R+r_{n}}=-D \sum_{\boldsymbol{x} \in N} f_{r r}(\boldsymbol{x}) \\
\sigma_{r \phi} & =D \sum_{n=0}^{\infty} s_{n} \frac{\cos (2 \phi) \cos \left(\phi-\theta_{n}\right)}{R+r_{n}}=D \sum_{\boldsymbol{x} \in N} f_{r \phi}(\boldsymbol{x}) \\
\sigma_{\phi \phi} & =D \sum_{n=0}^{\infty} s_{n} \frac{\cos \left(\phi-\theta_{n}\right) \sin (2 \phi)-\sin \left(\phi-\theta_{n}\right)}{R+r_{n}}=D \sum_{\boldsymbol{x} \in N} f_{\phi \phi}(\boldsymbol{x})
\end{aligned}
$$

where again we describe a marked point process $N=\boldsymbol{x}_{1}, \boldsymbol{x}_{2}, \ldots$, in this case with two marks: $s_{n}$, acting a random variable that takes values \pm 1 with equal probability $1 / 2$, and $\theta_{n}$, which is bounded by the crystallography of the material to take one of three values (see [56]): $54.7^{\circ}, 125.3^{\circ}, 0^{\circ}$ for FCC lattices, and $35.3^{\circ}, 144.7^{\circ}, 90^{\circ}$ for BCC lattices; we assume the three planes have the same probability $1 / 3$.

Thus, $\mathbb{M}=\{-1,1\} \times\left\{0^{\circ}, 54.7^{\circ}, 125.3^{\circ}\right\}$ or generally, $\mathbb{M}=\mathbb{M}_{s} \times \mathbb{M}_{\theta}=\{-1,1\} \times\left\{\theta_{a}, \theta_{b}, \theta_{c}\right\}$. Furthermore, $r_{n}$ and $\phi_{n}$ are independent random variables, forming a two dimensional spatial point process $N_{r \phi} \in \mathbb{R}^{2}$. We define the collective variable $\boldsymbol{x}=(s, r, \phi, \theta) \in \mathbb{R}^{2} \times \mathbb{M}$ so as to define the marked point process $N=N_{r \phi} \times \mathbb{M}=\left(s_{0}, r_{0}, \phi_{0}, \theta_{0}\right),\left(s_{1}, r_{1}, \phi_{1}, \theta_{1}\right), \ldots$, which induces the collective intensity measure $\Lambda\left(\mathbb{R}^{2} \times \mathbb{M}\right)=\nu\left(\mathbb{R}^{2}\right) \times \mu_{s}\left(\mathbb{M}_{s}\right) \times \mu_{\theta}\left(\mathbb{M}_{\theta}\right)$. 
We may apply the Campbell theorem to each of the stress field components, which shows their expectations to vanish due to the signature of mark $s$ :

$$
\begin{aligned}
& \bar{\sigma}_{r r}=-D \int_{\mathbb{R}^{2}} \nu(\mathrm{d} r \times \mathrm{d} s) \int_{\mathbb{M}_{s}} \mu_{\theta}(\mathrm{d} \theta) \int_{\mathbb{M}_{s}} f_{r r}(s, r, \phi, \theta)\left[\frac{1}{2} \delta(s+1)+\frac{1}{2} \delta(s-1)\right] \mathrm{d} s=0 \\
& \bar{\sigma}_{r \phi}=D \int_{\mathbb{R}^{2}} \nu(\mathrm{d} r \times \mathrm{d} s) \int_{\mathbb{M}_{s}} \mu_{\theta}(\mathrm{d} \theta) \int_{\mathbb{M}_{s}} f_{r \phi}(s, r, \phi, \theta)\left[\frac{1}{2} \delta(s+1)+\frac{1}{2} \delta(s-1)\right] \mathrm{d} s=0 \\
& \bar{\sigma}_{\phi \phi}=D \int_{\mathbb{R}^{2}} \nu(\mathrm{d} r \times \mathrm{d} s) \int_{\mathbb{M}_{s}} \mu_{\theta}(\mathrm{d} \theta) \int_{\mathbb{M}_{s}} f_{\phi \phi}(s, r, \phi, \theta)\left[\frac{1}{2} \delta(s+1)+\frac{1}{2} \delta(s-1)\right] \mathrm{d} s=0
\end{aligned}
$$

The variances, however, are unbounded:

$$
\operatorname{Var}\left[\sigma_{i j}\right]=\infty
$$

because $s_{n}^{2}=1 \forall n \in N$. This means that albeit the average stress state on the origin might be 0 , the actual stress state of any one realisation of the random cloud may take any value, rendering a strong statistical indeterminacy.

In this case, the indeterminacy has its origin in the construction of the random process itself, namely in that the sign of individual dislocations has been allocated in an entirely random fashion. This is because the underlying summation of the stress fields of dislocations of positive and negative sign is conditionally convergent. That is, the divergence of the variance is closely related to the way mass, i.e., dislocations of positive and negative sign, are distributed in the $2 \mathrm{D}$ plane. So far, no provisions have been made in this regard, other than that the sign of the dislocations be either positive or negative with equal probability. Arguably, this entails that there are on average the same number of positive and negative dislocations, i.e., that mass is conserved. However, in truth this is only conditionally so, since the mean signature is indeed zero:

$$
\bar{s}=E\left[\sum_{n=0}^{\infty} s_{n}\right]=\int_{\mathbb{R}} s\left[\frac{1}{2} \delta(s+1)+\frac{1}{2} \delta(s-1)\right] \mathrm{d} s=0
$$

but the variance is also unbounded

$$
\operatorname{Var}[s]=E\left[\left(\sum_{n=0}^{\infty} s_{n}\right)^{2}\right]=\sum_{n=0}^{\infty} \operatorname{Var}\left[s_{n}\right]=\sum_{n=0}^{\infty} 1=\infty
$$

To wit, albeit on average mass is conserved, any one realisation of the random point process so far assumed need not, and almost surely will not, conserve mass.

This suggests that a certain order needs to be imposed into how the positive and negative dislocations are arranged in the crystal; given the stochastic nature of the spatial process, there are many options in this regard. In studying random planar arrangements of dislocations in finite crystals, Wilkens [57] and Kocks and Scattergood [58] grouped dislocations into spatial sections containing the same number of dislocations (cf.[46]). The dislocations were randomly distributed within the section, and they were able to show that the averaged total elastic energy of such system was non-vanishing. Such construction would therefore serve to model areas of large net Burgers vectors that are balanced by steps at the free surface so as to conserve mass. The system under consideration here, in turn, is infinite, so the global mass conservation criterion can only be satisfied if the same number of positive and negative dislocations is guaranteed to exist in each realisation of the random process. In the following, we shall enforce this locally by considering not individual edge dislocations, but randomly located dipoles of edge dislocations instead; similar constructions where employed by Csikor and Groma [47] in computing the probability distribution of random populations of straight edge dislocations. This ensures that mass is locally conserved whilst providing enough flexibility that local areas of large net Burgers vectors may still be recovered by restricting the dipolar length or its orientation. Albeit this solution is not unique, it is motivated by two factors. On the one hand, it serves to represent bulk dislocation patterns such as those expected in Taylor's [51] model (see $[8,59]$ ). On the other hand, it draws a direct analogy with the three dimensional dislocation constructions that we shall be considering in section 4: the dipole would represent the cross-section of a dislocation loop with the plane.

\subsection{Mass conservation via dipolar moments}

In order to ensure that each realisation of the random process conserves mass, here we consider a random process where the individual events are not single edge dislocations any longer but dipoles of 


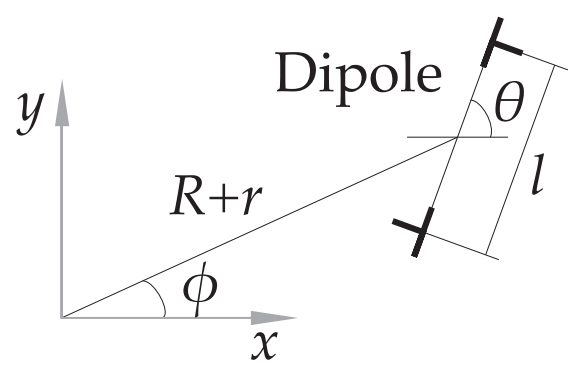

FIG. 1. Configuration of the two-dimensional system of dipoles of dislocations.

edge dislocations. In this planar case, the dipoles represent the necessary topological closure of the dislocation loop in three dimensions (see $[60,61]$ for how three dimensional loops correspond with planar dislocation dipoles). As said, this solution is not unique, and depending on the dislocation patterns under consideration, one could employ alternative constructions, such as for instance quadrupoles of dislocations (required for example in shock front models [62]), and then proceed as detailed below to compute the stress fields.

Therefore, here we must consider the fields due to a cloud of dipoles of dislocations. In the most general case, we shall consider a cloud of dipoles defined by the distance $l$. Inasmuch as the cloud is located by construction at sufficient distance from the origin, we can approximate this cloud of dipoles via their respective dipolar field expansions (see [50] and the Appendix), which renders the following stress field components in polar coordinates:

$$
\sigma_{r r}(r, \phi)=2 D l \frac{\sin (2 \phi)}{r^{2}}, \quad \sigma_{r \phi}(r, \phi)=-2 D l \frac{\cos (2 \phi)}{r^{2}}, \quad \sigma_{\phi \phi}(r, \phi)=0
$$

where in this case $D=\frac{B \mu(\lambda+\mu)}{\pi(\lambda+2 \mu)}$, with $\lambda$ and $\mu$ the first and second of Lamé's parameters. We note that the fields are expressed relative to the centre of the dipole, and aligned with its slip plane.

The configuration we are interested in here is the one shown in fig. 1: each dipole will be characterised by the position of its central point, which is determined via the same $N=\left\{\left(r_{n}, \phi_{n}\right)\right\}_{n=1}^{\infty}$ spatial point process, associated to which in this case we define the marks $\theta_{n} \in \mathbb{M}_{\theta}=\left\{\theta_{a}, \theta_{b}, \theta_{c}\right\}$, which describes the slip systems, the signature of the dipole $s_{n} \in \mathbb{M}_{s}=\{-1,1\}$, which describes the relative sign of one dislocation with respect to another, and $l_{n} \in \mathbb{M}_{l} \subset(0, \infty)$, which describes the separation distance between the dislocations forming the dipole, and which we shall assume below to either be a uniformly distributed random variable, or a log-normally distributed one.

The corresponding random variables of interest here (translated and rotated to align them with the original plane) are then

$$
\begin{aligned}
\sigma_{r r} & =D \sum_{n=0}^{\infty} s_{n} l_{n} \frac{\sin \left(2 \phi_{n}\right)+\sin \left(4 \phi_{n}-2 \theta_{n}\right)}{\left(R+r_{n}\right)^{2}}=D \sum_{\boldsymbol{x} \in N} f_{r r}(\boldsymbol{x}) \\
\sigma_{r \phi} & =-D \sum_{n=0}^{\infty} s_{n} l_{n} \frac{\cos \left(4 \phi_{n}-2 \theta_{n}\right)}{\left(R+r_{n}\right)^{2}}=-D \sum_{\boldsymbol{x} \in N} f_{r \phi}(\boldsymbol{x}) \\
\sigma_{\phi \phi} & =D \sum_{n=0}^{\infty} s_{n} l_{n} \frac{\sin \left(2 \phi_{n}\right)-\sin \left(4 \phi_{n}-2 \theta_{n}\right)}{\left(R+r_{n}\right)^{2}}=D \sum_{\boldsymbol{x} \in N} f_{\phi \phi}(\boldsymbol{x})
\end{aligned}
$$

The resulting point process is now marked by $s_{n}, l_{n}$, and $\theta_{n}$. As before, $s_{n}$ and $\theta_{n}$ follow a Rademacherlike distribution. In this case, however, $l_{n}$ is continuously distributed. On a first approach, we assume that $l_{i}$ is uniformly distributed with support over a positive interval $\left(l_{\min }, l_{\max }\right] \subset \mathbb{R}$, the bounds being given by the minimum and maximum lengths allowed.

a. Uniformly distributed dipole lengths. Before examining that case, however, we shall assume that $l_{n}$ is uniformly distributed with p.d.f. $\lambda(l)=1 /\left(l_{\max }-l_{\min }\right)$. In that case, we have that, invoking Campbell's theorem, we can only state that the average stress will be proportional to the average dipole distance, $\left(l_{\max }+l_{\min }\right) / 2$ :

$$
\bar{\sigma}_{i j}=2 D \int_{\mathbb{R}^{2}} \nu(\mathrm{d} r \times \mathrm{d} \phi) \int_{\mathbb{M}_{\theta}} \mu_{\theta}(\mathrm{d} \theta) \int_{\mathbb{M}_{s}} \mu_{s}(\mathrm{~d} s) \int_{\mathbb{M}_{l}} f_{i j}(\boldsymbol{x}) \frac{1}{l_{\max }-l_{\min }} \mathrm{d} l=D\left(l_{\max }+l_{\min }\right) \int_{\mathbb{R}^{2}} \nu(\mathrm{d} r \times \mathrm{d} \phi) \int_{\mathbb{M}_{\theta}} \frac{f_{i j}(\boldsymbol{x})}{l} \mu_{\theta}(\mathrm{d} \theta)
$$


In the most randomised case possible, it is reasonable to assume that the spatial point process is a stationary Poisson process, with $r$ and $\phi$ independent variables the intensity of which is characterised by Poisson parameters $\bar{r}$ and $\bar{\phi}$, whereupon the induced measure is $\nu(B)=\bar{r} \bar{\phi} \operatorname{Area}(B)$. The idea that dislocation microstructures may locally follow Poisson statistics has in the past been employed as a test case in a number of studies on the fractal nature of dislocation collectives [12,63, 64].

In that case, then, we have that the mean value of stress at the origin vanishes:

$$
\bar{\sigma}_{i j}=0 \quad \forall i, j
$$

due to the angular symmetry entailed by $\phi$.

The variance of the stress at the origin, in turn, is finite, and given by

$$
\begin{aligned}
\operatorname{Var}\left[\sigma_{r r}\right] & =\frac{\pi D^{2} \bar{\rho}_{\mathrm{dis}}}{12 R^{2}}\left(l_{\max }^{2}+l_{\text {max }} l_{\text {min }}+l_{\text {min }}^{2}\right) \\
\operatorname{Var}\left[\sigma_{r \phi}\right] & =\frac{\pi D^{2} \bar{\rho}_{\text {dis }}}{24 R^{2}}\left(l_{\text {max }}^{2}+l_{\text {max }} l_{\text {min }}+l_{\text {min }}^{2}\right) \\
\operatorname{Var}\left[\sigma_{\phi \phi}\right] & =\frac{\pi D^{2} \bar{\rho}_{\text {dis }}}{12 R^{2}}\left(l_{\max }^{2}+l_{\text {max }} l_{\text {min }}+l_{\text {min }}^{2}\right)
\end{aligned}
$$

where $\bar{\rho}_{\text {dis }}$ is the average dislocation density.

The average dislocation density is, in effect, twice the average intensity of the process, hereafter $\bar{\Lambda}$, since the latter measures the average number of dislocation dipoles per unit area. It is important to note that, despite the population being largely disordered, in this study we maintain the Taylor style scaling between the average distance between dipoles, $\bar{x}$, and the (average) dislocation density, $\bar{\rho}_{\text {dis }}$, as $\bar{x} \propto \bar{\rho}_{\text {dis }}^{-1 / 2}$ [51]. Indeed, the average distance may be computed from the nearest neighbour function (a cumulative distribution function), which for a Poisson process is simply, by Rényi's theorem[65]

$$
D(r, \phi)=1-e^{-\bar{\Lambda} \pi r^{2}}
$$

Then, the average distance $\bar{x}$ between dipoles is

$$
\bar{x}=\frac{1}{2 \pi} \int_{0}^{2 \pi} \mathrm{d} \phi \int_{0}^{\infty} \phi r\left(2 \pi \bar{\Lambda} r e^{-\bar{\Lambda} \pi r^{2}}\right) \mathrm{d} r=\frac{\pi}{2 \sqrt{\bar{\Lambda}}}=\frac{\pi}{2 \sqrt{2} \sqrt{\bar{\rho}_{\mathrm{dis}}}}
$$

Thus, the average distance between particles remains proportional to $\bar{\rho}_{\mathrm{dis}}^{-1 / 2}$, even if clearly the internal disorder entails plastic neutrality.

b. Non-uniformly distributed dipole lengths. In the event $l_{n}$ is non-uniformly distributed over $\mathbb{R}^{+}$, then it most likely adopts a log-normal distribution, as was argued by Shishvan and Van der Giessen [60] when discussing the length of a Frank-Read source. This assumes that the dipole length is never negative, and that its most usual values are concentrated about its mean, by making the logarithm of $l$ be normally distributed. The ensuing mark's support is then $\mathbb{R}^{+}=(0, \infty)$. We define the mean and standard deviation of the underlying log-normal distribution in terms of the admissible $l_{\min }$ and $l_{\max }$ defined by the minimum and maximum allowable distance in a dipole. Here, we set $l_{\min }$ as the distance below which the mutually attractive stress is the ideal lattice strength $\mu /(4 \pi)[66]$, which is given by $l_{\min }=\frac{2 B}{1-\nu}$ (see [9]). We set $l_{\max }$ as defined by the system's likely grain geometry: $l_{\max }=\sqrt{D}$ for $D$ the grain size. Let $m$ and $\varsigma$ denote the mean and the standard deviation of the associated normal distribution. We set their values by imposing a $3-\Sigma$ criterion as done in [60], so that

$$
\begin{aligned}
& m+3 \varsigma=\ln l_{\max } \\
& m-3 \varsigma=\ln l_{\min }
\end{aligned}
$$

whereupon

$$
m=\ln \sqrt{l_{\max } l_{\min }}, \quad \varsigma=\ln \left(\frac{l_{\max }}{l_{\min }}\right)^{\frac{1}{6}}
$$

The resulting probability distribution function of lengths is then

$$
f_{l}(l)=\frac{1}{l \varsigma \sqrt{2 \pi}} e^{-\frac{(\ln l-m)^{2}}{2 \varsigma^{2}}}
$$

for $m$ and $s$ defined as above. 


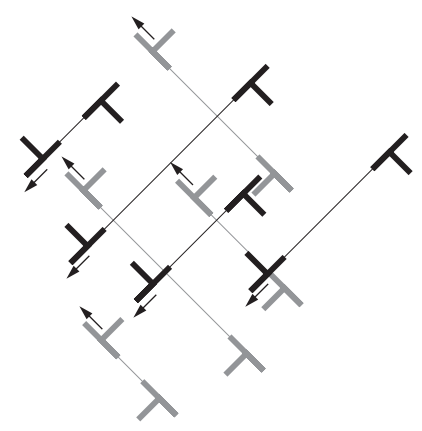

FIG. 2. A random population of aligned dipoles. Note the local net Burgers vector they induce.

The resulting average is again $\bar{\sigma}_{i j}=0$ due to the angular symmetry about the origin, but the variance is different, albeit still finite:

$$
\begin{aligned}
\operatorname{Var}\left[\sigma_{r r}\right] & =\frac{\pi D^{2} \bar{\rho}_{\mathrm{dis}} e^{2 m+2 \varsigma^{2}}}{R^{2}} \\
\operatorname{Var}\left[\sigma_{r \phi}\right] & =\frac{\pi D^{2} \bar{\rho}_{\mathrm{dis}} e^{2 m+2 \varsigma^{2}}}{2 R^{2}} \\
\operatorname{Var}\left[\sigma_{\phi \phi}\right] & =\frac{\pi D^{2} \bar{\rho}_{\mathrm{dis}} e^{2 m+2 \varsigma^{2}}}{R^{2}}
\end{aligned}
$$

This shows that so long as the distribution of dipole lengths remains independent from their geometric location, the effective stress of a random cloud of dislocations on a certain point is zero, but that the variance found between different realisations is finite. It also confirms that a material cannot be relaxed by a disordered distribution of dislocations, but that there is a statistical possibility that it be shielded (or, unshielded) with standard deviation proportional to $\sqrt{\bar{\rho}_{\text {dis }}}$, even despite the fact that the inherent scaling of the problem, i.e., the average distance between dipoles, scales with $1 / \sqrt{\bar{\rho}_{\text {dis }}}$.

\subsection{Partial orders: Taylor's equation and the Hall-Petch relation}

The direct implication of the analysis so far is that one can only expect a random collective of dislocations to exert a non-zero mean stress if additional ordering beyond mass conservation is enforced. This means that a given dislocation density is not sufficient to ensure plastic relaxation: one needs to provide the density field with some order. Hitherto, we have assumed that the population of dislocations is entirely random, both in each dislocation's location and in its attributes (e.g., its sign or slip plane).

The results presented so far crucially depend on mass conservation, and on the net neutrality of the signature or alignment of dislocation dipoles. This immediately raises the question of whether there are no random dislocation arrangements for which plastic relaxation may be possible. As we show in this section, they do indeed exist, but must be achieved by inducing an additional, partial order on the random collective of dislocations. In this section we shall study two such partial orders: alignment of the dipoles, and spatial localisation.

By dipole alignment we refer to providing all dipoles with the same signature, leading to, locally, regions of the same net Burgers vector - note however that the overall net Burgers vector of each dipole is zero, but that in aligning dipoles we may generate regions of large effective Burgers vector, as we indicate in fig.2. By spatial localisation, we mean localising the dislocation into specific regions of space, but without surrendering the underlying randomness of a point process. We shall achieve this by modifying the intensity of the point process to make it larger on specific regions of space.

\subsubsection{Biasing the dipolar alignment: Taylor's equation.}

In section 33.1 , the alignments of dipoles was treated as a stochastic variable via the signature $s_{n}$, and by allowing $2 \pi$ rotations about the directions of slip. In this section, we wish to induce a partial order in the random cloud of dislocations such that all dipoles are aligned in such a way as to induce a long range stress field of the same signature.

There are a number of relevant physical situations that may lead to such partially ordered ensembles of dislocations. An example may be found in shock loading models such as the Smith-Hornbogen interface 
$[67,68]$ or in the Meyers model of a shock front $[62,69]$ : in order to accommodate the vast lattice compression induced by the shock front, both models postulate an interface of aligned dipoles of dislocations; albeit mass is conserved, the shock front is shielded by the dislocations of the same signature, whilst those of opposite sign move away from the front and are left behind. Hence, an effective region of large net Burgers vector is created locally about the front, which is accordingly relaxed. Still, we have seen any such dipole induces a long-range, net stress field (given by eqn.14 in the dipolar field expansion), and in this case we are interested in studying the effect ordering the dipoles in such a way that the net stress field is of the same sign on the origin.

A situation reminiscent of this is also found in the notion of arrays of geometrically necessary dislocations, and their apparent role in plastic relaxation [70,71]. Furthermore, ordered dipoles of dislocations are commonly observed in dicrete dislocation simulations, where they arise to relax the external stress fields. Thus, they may be found in the study of beams that are plastically relaxed while subjected to a flexile load: when studying thin foils of material subjected to simple bending loads, Cleveringa et al.[72] report that dislocation dipoles nucleated from Frank-Read sources minimise the external, constant stress applied on the Frank-Read source, resulting in configurations of dipoles of the same alignment. Other situations leading to the alignment of the dipoles may be found in [27], where the authors report similar structures for thin strips subjected to tensile static loads; in [73], where Balint et al. examined the way dislocation sources give raise to the Hall-Petch effect in polycrystalline thin foils; or in [33], where the dipoles are equally aligned so as to consistently shield a shock front. Albeit in these cases the net Burgers vector is zero (so as to preserve mass), the dipoles align themselves so as to contribute to the relaxation.

In order to achieve a partial order where all dipoles are equally aligned, we may simply model the signature of the fields in eqn.15 to be a sign function $s(\phi)$ such that it flips the sign of the field each quadrant of $\phi$, rather than as a mark associated with the spatial Poisson process. We may express this as:

$$
s(\phi)=\operatorname{sgn}\left[\sin \frac{\pi \phi}{2}\right]
$$

In effect this would align all dipoles in this specific way about the epicentral line.

If we multiply each field component in eqn.15 and proceed as before, we find the following mean values of stress at the origin to be, for the case when $l_{n}$ is uniformly distributed:

$$
\bar{\sigma}_{r r}=D \bar{l} \bar{\rho}_{\text {dis }} \ln \left(\frac{R+r_{\max }}{2 R}\right)=\bar{\sigma}_{\phi \phi}, \quad \bar{\sigma}_{r \phi}=0
$$

where $r_{\text {max }}$ denotes the maximum value of $r$ and $\bar{l}$ the average dipolar length. Clearly, if $r \rightarrow \infty, \bar{\sigma}_{r r}$ and $\bar{\sigma}_{\phi \phi}$ diverge, which is to be expected because in this partial order all dipoles contribute with the same sign to the relaxation of the origin; unless there is a physical limitation to the spatial distribution of these dipoles, the stress fields will diverge. The associated variances are:

$$
\begin{aligned}
\operatorname{Var}\left[\sigma_{r r}\right] & =\frac{\pi D^{2} \rho_{\text {dis }} r_{\text {max }}\left(l_{\text {max }}^{2}+l_{\text {max }} l_{\text {min }}+l_{\text {min }}^{2}\right)\left(2 R+r_{\text {max }}\right)}{12 R^{4}} \\
\operatorname{Var}\left[\sigma_{r \phi}\right] & =\frac{\pi D^{2} \rho_{\text {dis }} r_{\text {max }}\left(l_{\text {max }}^{2}+l_{\text {max }} l_{\text {min }}+l_{\text {min }}^{2}\right)\left(2 R+r_{\text {max }}\right)}{24 R^{4}} \\
\operatorname{Var}\left[\sigma_{\phi \phi}\right] & =\frac{\pi D^{2} \rho_{\text {dis }} r_{\text {max }}\left(l_{\text {max }}^{2}+l_{\text {max }} l_{\text {min }}+l_{\text {min }}^{2}\right)\left(2 R+r_{\text {max }}\right)}{12 R^{4}}
\end{aligned}
$$

It is significant that in imposing this partial order, the crystallographical alignment of the dipoles becomes irrelevant when computing the mean and the variance; this is because the alignment makes the contributions to the stress state consistently of the same sign for all dipoles. We also note that the coefficient of variation (i.e., the ratio between the standard deviation and the mean of the stress field) is necessary smaller than one, which suggests a small variability in the actual stress field between different realisations of the cloud of dislocation dipoles.

We observe two apparent physical limits the net effect this population of dislocations may have on a material point: (1) the effective stress field will be directly proportional to that population's dislocation density, $\bar{\sigma} \propto \bar{\rho}_{\text {dis }}$, irrespective of the values of $R$ and $r_{\max } ;(2)$ the magnitude of the shielding in this case will be dominated by the average dipolar length, which will most likely be considerably smaller than the grain size, i.e., $\bar{l} \ll \sqrt{A_{\text {grain }}}$. If $\bar{l}$ is uncorrelated to any other physical feature, then the scaling of $\bar{\sigma}_{i j}$ will be proportional to $\bar{\rho}_{\text {dis }}$. However, if we allow $\bar{l} \approx \bar{x}$, we recover Taylor's equation: $\bar{\sigma}_{i j} \propto \sqrt{\bar{\rho}_{\text {dis }}} \delta_{i j}$. This assumption underlies most of Taylor's own [51] and subsequent analyses (see for instance [74] and [59],pp. 9-10.), as is a crucial requirement for the formation of a lattice of dislocations that is in mechanical equilibrium. 


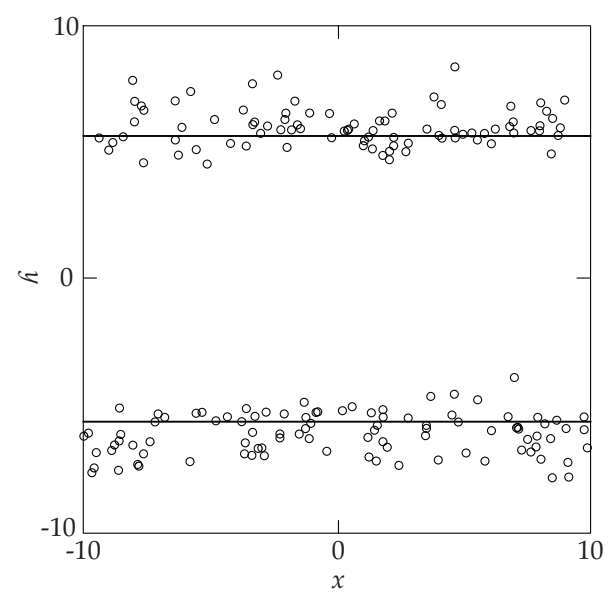

FIG. 3. Simulation of the spatial locations of a inhomogeneous distribution of dislocation dipoles given by eqn.28. Here $s=0.1, m=\sqrt{3}, \bar{\nu}=100$. Here each point would represent a dipole of edge dislocations.

Thus, in recovering Taylor's equation, we require that the average size of a dipole be approximately equal to the average distance between dipoles. We need not assume (and indeed in taking a Poisson process, we have implicitly assumed the opposite), that the specific dislocations forming the stochastic cloud interact with one another; in using a point process we merely require a statistical description of the distribution of dipolar lengths and of the intensity of the spatial point process. However, $\bar{l} \approx \bar{x}$ implicitly assumes that mechanical equilibrium between the different dislocations in the dipole has been established.

We note that eqn.26 may be used to estimate the degree of disorder in a material: if Taylor's scaling breaks down, as it happens for instance with the Bauschinger effect after a few cycles of loading in fatigue, when the dislocation density keeps increasing but the yield point remains constant [75, 76], in light of eqn.26 and the previous section one may surmise that the newly generated population of dipoles are either much closely packed than their average distance (i.e., $\bar{l} \ll \bar{x}$ ), or that it is simply heavily disordered, and their additional collective effect is plastically neutral. In other words, we find two lengthscales at play: the one related to the spacing between dislocation dipoles, which governs the dislocation density, and the one related to the dipolar distance, which governs the internal arrangement of dislocations. Only if they both match is Taylor's equation obeyed.

An additional consideration may be drawn from eqn.26, in this case for discrete dislocation simulations of plasticity, where it is often the case that a random population of dislocations is used as a seed for studying their posterior evolution [9,77]. If the initial assumption is that the material to simulate has been deformed or otherwise should be expected to display Taylor-style hardening, then the initial population of random dislocations ought to be chosen in such a way as to ensure that the average dipolar length scales with the initial dislocation density.

\subsubsection{Non uniform spatial distribution of dislocation dipoles: the Hall-Petch equation.}

So far, we have assumed that the dipoles of dislocations remain homogeneously distributed about the origin with constant intensity measure. An alternative way of inducing a partial order in the population of dislocations would consist on inducing a greater degree of spatial localisation on the cloud of dislocations. This would align with the notion of a forest of dislocations [59] or, otherwise, dislocation walls, lamellar structures and similar such arrangements (cf. [8, 78]), which we may therefore hope to model by considering spatially localised, random populations of dislocations. Although it is arguably inaccurate to assume that the dislocations in a forest are distributed entirely at random, it is a typical assumption in many models [79].

Thus, let us assume that the population of dislocation dipoles is concentrated about a certain band at some average distance $\bar{y}$ from the origin. We may represent ex hypothesi the Poisson process' intensity with the mathematical form of pseudo-log normal distribution about

$$
\nu(\boldsymbol{x})=\frac{\bar{\nu}}{2 \sqrt{2 \pi} s|y|} e^{-\frac{(\ln |y|-m)^{2}}{2 s^{2}}}
$$

where $m$ is the average distance of the band to the origin, and $s$ an adjustable parameter - the standard 
deviation of the underlying log-normal intensity - and $\bar{\nu}$ some mean intensity. Accordingly, we are considering a spatial distribution of dislocation dipoles similar to the one shown in fig.3, which concentrates the intensity measure about two bands (the narrowness of which is set by $s$ ) parallel to the $x$ axis. Thus we are able to reproduce a wall-like structure of random dislocations, and retain the randomness of the spatial distribution of points.

The average stress in this case may still be computed invoking Campbell's theorem, which holds for inhomogeneous Poisson processes. We shall assume that the dipolar lengths are uniformly distributed. This renders ${ }^{2}$

$$
\bar{\sigma}_{i j}=D \bar{l} \bar{\nu} e^{\left(-2 m+2 s^{2}\right)} \kappa_{i j}\left(\theta_{a}, \theta_{b}, \theta_{c}\right)
$$

where $\kappa_{i j}$ is a geometric factor (not reproduced here due to its length), and $\bar{l}$ the average dipolar length. The introduction of an alignment factor in the dipoles only modifies the form of the $\kappa_{i j}$ functions. In this case the dislocation density is largely localised along the two bands of dislocations; however, the average dislocation density remains the intensity measure of the process,

$$
\bar{\rho}_{\text {dis }}=\int_{\mathbb{R} \times \mathbb{R}} \frac{\bar{\nu}}{2 \sqrt{2 \pi} s|y|} e^{-\frac{(\ln |y|-m)^{2}}{2 s^{2}}} \mathrm{~d} x \mathrm{~d} y=\bar{\nu} e^{\left(m+\frac{s^{2}}{2}\right)}
$$

whereupon

$$
\bar{\sigma}_{i j}=D \bar{l} \bar{\rho}_{\mathrm{dis}} e^{-\frac{m}{2}} e^{\frac{3 s^{2}}{2}} \kappa_{i j}\left(\theta_{a}, \theta_{b}, \theta_{c}\right)
$$

Two things stand out from this result. On the one hand, without having previously assumed any property related to the spatial alignment of dipoles, we reach with eqn.31 an equation reminiscent of eqn.26. As before, we may argue that if $\bar{l} \approx \bar{x}$ (and in this case $\bar{x} \propto 1 / \sqrt{\rho_{\mathrm{dis}}}$ as well as per properties of Poisson processes [65]), then $\bar{\sigma}_{i j} \propto \sqrt{\bar{\rho}_{\text {dis }}}$, so that heavily localised random distributions of dislocations of the sort that are observed in Kuhlmann-Wilsdorff style cell structures [78, 80] or in lamellar cell structures $[81,82]$ contribute to the hardening of the material with a Taylor-style law, even if the underlying microstructure may be regarded as largely random. At the same time, we must point out that if $\bar{l}$ is not related to the average distance between dislocations $\bar{x}$, then Taylor's equation need not be obeyed by the specific population of dislocations.

On the other hand, we note that eqn.31 is directly proportional to $e^{-m / 2}$, to wit, the inverse of (half) the geometric mean distance between the two bands of dislocations. Thus, if the median distance between the two 'walls' is $d$, then eqn.31 predicts $\bar{\sigma}_{i j}$ be inversely proportional to $d$. If such median distance were to be regarded proportional to the grain size, i.e., if $d \propto \sqrt{D}$ (this is reasonable if the dislocations are clustered in lamellar structures), then eqn.31 would scale according to the Hall-Petch equation (cf.[83]). We also note that $\bar{\sigma}_{i j}$ is directly influenced by the width of the wall via the $e^{\frac{3 s^{2}}{2}}$ factor.

Thus, by considering a simple wall-like random structure of dislocations, we are able to recover both the Hall-Petch and Taylor's equations. Future work will focus on exploring such relations between cell sizes and clustered random clouds of dislocations.

\section{PLASTIC RELAXATION INDUCED BY RANDOM CLOUDS OF DISLOCATIONS IN THREE DIMENSIONS}

The $3 \mathrm{D}$ case may in some ways be regarded as a generalisation of the $2 \mathrm{D}$ case. The main difference lies in the nature of the distribution of the random cloud dislocations itself, which in this case must be formed by dislocation loops of different shapes and sizes. As before, we assume that the cloud of now dislocation loops lies at sufficient distance from the origin that their effect may be substituted by their force multipolar expansions, as given in [50]. The cloud is then subsumed to a set of points in $\mathbb{R}^{3}$ which we distribute stochastically via a marked Poisson process. The corresponding stress fields can then be integrated stochastically via Campbell's theorem. In the $3 \mathrm{D}$ case particular care must be taken in orienting the dislocation loops according to an appropriate crystallographic orientation, which is detailed in the following discussion. Although they made no attempt to compute the stress field, El Azab et al.[48] proposed modelling 3D dislocation networks using stochastic fibre processes, which may be regarded as a generalisation of the point processes approach we adopt here (see [84]). In a fibre process, rather than

\footnotetext{
2 If rather than two disperse walls of dislocations, as we have considered above, we were to have only one (which may be achieved by exchanging $|y|$ for $y$ in eqn.28), one case prove that $\bar{\sigma}_{i j}$ is half the one predicted by eqn.29.
} 
describing the distribution of dislocations as a spatial distribution of individual points, the dislocations lines are treated explicitly. Albeit more accurate in describing the network of dislocations, the evaluation of the stress fields associated with a fibre process would be considerably more expensive, particularly since Campbell's theorem does not generally apply[55]. The description of the dislocation loops via their multipolar field expansions, albeit an approximation, enables a simpler approach at computing the loop's long range contribution. This facilitates the evaluation of the stress field averages of complex networks at a fraction of the computational cost. As will be seen, employing higher order corrections to the force multipolar field expansion produces an increasingly accurate approximation to the short range stress fields.

\subsection{Dipolar expansion of a dislocation loop}

We shall assume the loops are planar, and to begin with approximate their fields up to dipolar order. In an elastic isotropic medium, the dipolar moment $\gamma_{p k}^{(1)}$ of a planar dislocation loop of area $A_{l}$ is given by $[50]$

$$
\gamma_{p k}^{(1)}=B_{i} \nu_{j}\left[\lambda \delta_{i j} \delta_{p k}+\mu\left(\delta_{i p} \delta_{j k}+\delta_{i k} \delta_{j p}\right)\right] A_{l}
$$

where $B_{i}$ is the Burgers vector, $\nu_{j}$ the slip plane normal, $\mu$ the shear modulus and $\lambda$ Lamé's first parameter. We set an orthonormal basis at the centre of area of the loop. The $x_{1}, x_{2}$ plane contains the loop, and the loop's normal is parallel to the $x_{3}$ direction. Hence, in the most general case $B_{i}=\left[B_{1} B_{2} 0\right]^{T}$ is the Burgers vector and $\nu_{j}=\left[\begin{array}{lll}0 & 0 & 1\end{array}\right]^{t}$. Relative to these axes, we find that the only non-zero components of $\gamma_{p k}^{(1)}$ are:

$$
\gamma_{13}^{(1)}=\gamma_{31}^{(1)}=B_{1} \mu A_{l}, \quad \gamma_{23}^{(1)}=\gamma_{32}^{(1)}=B_{2} \mu A_{l}
$$

Therefore, the multipolar expansion is given by

$$
u_{i}(\boldsymbol{x})=\left[\frac{\partial G_{i 1}}{\partial x_{3}}+\frac{\partial G_{i 3}}{\partial x_{1}}\right] B_{1} \mu A_{l}+\left[\frac{\partial G_{i 2}}{\partial x_{3}}+\frac{\partial G_{i 3}}{\partial x_{2}}\right] B_{2} \mu A_{l}
$$

where $G_{i j}$ is the $3 \mathrm{D}$ elastic isotropic Green's function [85],

$$
G_{i j}(\boldsymbol{x})=\frac{1}{8 \pi \mu} \frac{1}{r}\left[2 \delta_{i j}-\frac{\lambda+\mu}{\lambda+2 \mu}\left(\delta_{i j}-\frac{x_{i} x_{j}}{r^{2}}\right)\right], \quad r=\sqrt{x_{1}^{2}+x_{2}^{2}+x_{3}^{2}}
$$

The corresponding dipolar stress fields are collected in table I.

a. Higher order multipolar terms. In some circumstances, it might be of interest to consider the stress field contributions of higher order terms in the multipolar expansion as a means for obtaining better approximations of the short range fields of the dislocation loops. If, as we have defined it above, the local coordinate system is located on the centre of area (centroid) of the loop, then the second order 'quadrupolar' terms vanish because the first moments of area are all identically zero, and the next higher order term would be the 'octopolar' third order terms, dependent on the second moments of area. For simplicity, but without loss of generality, in subsequent discussions we shall assume that the loop is planar, and that therefore the displacement field contribution of the octopolar terms is of the form [50]

$$
u_{i}^{(3)}=\frac{1}{3 !}\left[3 \frac{\partial^{3} G_{i 1}}{\partial x_{3} \partial x_{1}^{2}} \gamma_{1311}^{(3)}+3 \frac{\partial^{3} G_{i 1}}{\partial x_{3} \partial x_{2}^{2}} \gamma_{1322}^{(3)}+3 \frac{\partial^{3} G_{i 1}}{\partial x_{3} \partial x_{3}^{2}} \gamma_{3122}^{(3)}+3 \frac{\partial^{3} G_{i 3}}{\partial x_{1}^{3}} \gamma_{3111}^{(3)}\right]
$$

The corresponding stress field contributions may be obtained from differentiation, as $\sigma_{i j}=\lambda \delta_{i j} u_{k, k}+$ $\left.\mu u_{i, j}\right)$.

The octopole moments $\gamma_{p k_{1} k_{2} k_{3}}^{(3)}$ depend on the second moment of area of the loop

$$
\gamma_{p k_{1} k_{2} k_{3}}^{(3)}=B_{i} \nu_{j}\left(\lambda \delta_{i j} \delta_{p q}+\mu\left(\delta_{i p} \delta j q+\delta_{i q} \delta_{j p}\right)\right) \sum_{i=1}^{3} \delta_{k_{i} q} \prod_{j=1, j \neq i}^{3} J_{u_{1} u_{2}}
$$

where

$$
J_{u_{1} u_{2}}=\int_{D} x_{u_{1}} x_{u_{2}} \mathrm{~d} D
$$




\begin{tabular}{|c|c|}
\hline Stress component & Dipolar expansion \\
\hline$\sigma_{11}$ & $\begin{array}{l}\frac{-3 A_{l} x_{3}}{8 \pi(\lambda+2 \mu) r^{7}}\left[B_{2}\left(\lambda^{2}\left(x_{1}^{3}-3 x_{1}^{2} x_{2}-4 x_{1} x_{2}^{2}+x_{1} x_{3}^{2}+2 x_{2}^{3}-3 x_{2} x_{3}^{2}\right)+\right.\right. \\
\left.+\lambda \mu\left(x_{1}^{3}-23 x_{1}^{2} x_{2}-4 x_{1} x_{2}^{2}+x_{1} x_{3}^{2}+2 x_{2}^{3}-3 x_{2} x_{3}^{2}\right)+4 \mu^{2} x_{2}\left(-4 x_{1}^{2}+x_{2}^{2}+x_{3}^{2}\right)\right)+ \\
+B_{1}\left(\lambda^{2}\left(-\left(x_{1}-x_{2}\right)\right)\left(x_{1}^{2}+5 x_{1} x_{2}+x_{2}^{2}+x_{3}^{2}\right)+\right. \\
\left.\left.+\lambda \mu\left(-17 x_{1}^{3}-4 x_{1}^{2} x_{2}+8 x_{1} x_{2}^{2}+3 x_{1} x_{3}^{2}+x_{2}^{3}+x_{2} x_{3}^{2}\right)+4 \mu^{2} x_{1}\left(-4 x_{1}^{2}+x_{2}^{2}+x_{3}^{2}\right)\right)\right]\end{array}$ \\
\hline$\sigma_{22}$ & $\begin{array}{l}\frac{3 A_{l} x_{3}}{8 \pi(\lambda+2 \mu) r^{7}}\left\{B_{1}\left[\lambda^{2}\left(x_{1}-x_{2}\right)\left(x_{1}^{2}+5 x_{1} x_{2}+x_{2}^{2}+x_{3}^{2}\right)+\right.\right. \\
\left.+\lambda \mu\left(3 x_{1}^{3}+12 x_{1}^{2} x_{2}+x_{1}\left(8 x_{2}^{2}+3 x_{3}^{2}\right)-3 x_{2}\left(x_{2}^{2}+x_{3}^{2}\right)\right)-2 \mu^{2}\left(x_{1}+x_{2}\right)\left(x_{1}^{2}-5 x_{1} x_{2}+x_{2}^{2}+x_{3}^{2}\right)\right]+ \\
+B_{2}\left[-\lambda^{2}\left(x_{1}^{3}-3 x_{1}^{2} x_{2}-4 x_{1} x_{2}^{2}+x_{1} x_{3}^{2}+2 x_{2}^{3}-3 x_{2} x_{3}^{2}\right)+\right. \\
\lambda \mu\left(-3 x_{1}^{3}+5 x_{1}^{2} x_{2}+12 x_{1} x_{2}^{2}-3 x_{1} x_{3}^{2}+10 x_{2}^{3}+5 x_{2} x_{3}^{2}\right)+ \\
\left.\left.+2 \mu^{2}\left(-x_{1}^{3}+x_{1}^{2} x_{2}+4 x_{1} x_{2}^{2}-x_{1} x_{3}^{2}+6 x_{2}^{3}+x_{2} x_{3}^{2}\right)\right]\right\}\end{array}$ \\
\hline$\sigma_{33}$ & $\begin{array}{l}\frac{3 A_{l} x_{3}(\lambda+\mu)}{8 \pi(\lambda+2 \mu) r^{7}}\left(B_{1} \lambda\left(x_{1}-x_{2}\right)\left(x_{1}^{2}+5 x_{1} x_{2}+x_{2}^{2}+x_{3}^{2}\right)-4 B_{1} \mu x_{1}\left(x_{1}^{2}+x_{2}^{2}-4 x_{3}^{2}\right)-\right. \\
\left.-B_{2} \lambda\left(x_{1}^{3}-3 x_{1}^{2} x_{2}+x_{1}\left(x_{3}^{2}-4 x_{2}^{2}\right)+2 x_{2}^{3}-3 x_{2} x_{3}^{2}\right)-4 B_{2} \mu x_{2}\left(x_{1}^{2}+x_{2}^{2}-4 x_{3}^{2}\right)\right)\end{array}$ \\
\hline$\sigma_{12}$ & $\begin{array}{l}-\frac{3 A_{l} \mu x_{3}}{8 \pi(\lambda+2 \mu) r^{7}}\left(B_{1} \lambda\left(-2 x_{1}^{3}-14 x_{1}^{2} x_{2}+3 x_{1}\left(x_{2}^{2}+x_{3}^{2}\right)+x_{2}\left(x_{2}^{2}+x_{3}^{2}\right)\right)-\right. \\
-B_{1} \mu\left(2 x_{1}^{3}+16 x_{1}^{2} x_{2}-3 x_{1}\left(x_{2}^{2}+x_{3}^{2}\right)+x_{2}\left(x_{2}^{2}+x_{3}^{2}\right)\right)+ \\
+B_{2} \lambda\left(x_{1}^{3}-4 x_{1}^{2} x_{2}-14 x_{1} x_{2}^{2}+x_{1} x_{3}^{2}+x_{2}^{3}+x_{2} x_{3}^{2}\right)- \\
\left.-B_{2} \mu\left(x_{1}^{3}+4 x_{1}^{2} x_{2}+x_{1}\left(16 x_{2}^{2}+x_{3}^{2}\right)-x_{2}\left(x_{2}^{2}+x_{3}^{2}\right)\right)\right)\end{array}$ \\
\hline$\sigma_{13}$ & $\begin{array}{l}-\frac{A_{l} \mu}{4 \pi(\lambda+2 \mu) r^{7}}\left(B_{1}\left(3 \lambda\left(x_{1}^{4}+x_{1}^{2}\left(x_{2}^{2}-8 x_{3}^{2}\right)+x_{3}^{2}\left(x_{2}^{2}+x_{3}^{2}\right)\right)+\right.\right. \\
\left.\left.+2 \mu\left(x_{1}^{4}+x_{1}^{2}\left(2 x_{2}^{2}-13 x_{3}^{2}\right)+\left(x_{2}^{2}+x_{3}^{2}\right)^{2}\right)\right)+3 B_{2} x_{1} x_{2}\left(\lambda\left(x_{1}^{2}+x_{2}^{2}-9 x_{3}^{2}\right)-10 \mu x_{3}^{2}\right)\right)\end{array}$ \\
\hline$\sigma_{23}$ & $\begin{array}{l}-\frac{A_{l} \mu}{8 \pi(\lambda+2 \mu) r^{7}}\left(B_{1} \lambda\left(2 x_{1}^{4}+3 x_{1}^{3} x_{2}+x_{1}^{2}\left(x_{2}^{2}-11 x_{3}^{2}\right)+3 x_{1}\left(x_{2}^{3}-14 x_{2} x_{3}^{2}\right)-x_{2}^{4}+x_{2}^{2} x_{3}^{2}+2 x_{3}^{4}\right)+\right. \\
+B_{1} \mu\left(2 x_{1}^{4}-3 x_{1}^{3} x_{2}+x_{1}^{2}\left(x_{2}^{2}-11 x_{3}^{2}\right)-3 x_{1}\left(x_{2}^{3}+16 x_{2} x_{3}^{2}\right)-x_{2}^{4}+x_{2}^{2} x_{3}^{2}+2 x_{3}^{4}\right)+ \\
+B_{2} \lambda\left(x_{1}^{4}+3 x_{1}^{3} x_{2}+5 x_{1}^{2}\left(x_{2}^{2}+x_{3}^{2}\right)+3 x_{1}\left(x_{2}^{3}-4 x_{2} x_{3}^{2}\right)+4 x_{2}^{4}-37 x_{2}^{2} x_{3}^{2}+4 x_{3}^{4}\right)+ \\
\left.+B_{2} \mu\left(5 x_{1}^{4}+3 x_{1}^{3} x_{2}+7 x_{1}^{2}\left(x_{2}^{2}+x_{3}^{2}\right)+3 x_{1}\left(x_{2}^{3}-4 x_{2} x_{3}^{2}\right)+2 x_{2}^{4}-41 x_{2}^{2} x_{3}^{2}+2 x_{3}^{4}\right)\right)\end{array}$ \\
\hline
\end{tabular}

TABLE I. Force dipole expansion of the stress field of a planar dislocation loop.

are the second moments of area of the loop, with $D$ the loop's area.

Since it depends on the second moment of area as well as on the loop's area, the octopolar approximation will capture with greater accuracy the actual shape of the loop. In accordance with the ansatz that the shape of the loops is a random variable, we note that if $\chi_{D}(\boldsymbol{x})$ is the characteristic function the support of which is the dislocation loop, then we may regard it as a probability measure over $D$ (the loop), whereby the second moments of area are statistical moments as well, i.e., $J_{u_{1} u_{2}}=E\left[u_{1} u_{2}\right]=\operatorname{Cov}\left(u_{1}, u_{2}\right)$, measuring the loop's shape covariances because the first moments vanish for the coordinate system located on the loop's centroid.

Given that the multipolar approximation is linear, the octopolar stress field can be regarded as the sum of the dipolar expansion and the octopolar terms described above, i.e., the full octopolar fields will be $\sigma_{i j}=\sigma_{i j}^{(1)}+\sigma_{i j}^{(3)}$. The form of $\sigma_{i j}^{3}$ is collected in the Appendix for the case of a planar loop. Similar expressions may be obtained for higher order multipolar terms (for more details, see [50]), and may be useful if greater accuracy in the short range fields is necessary. 


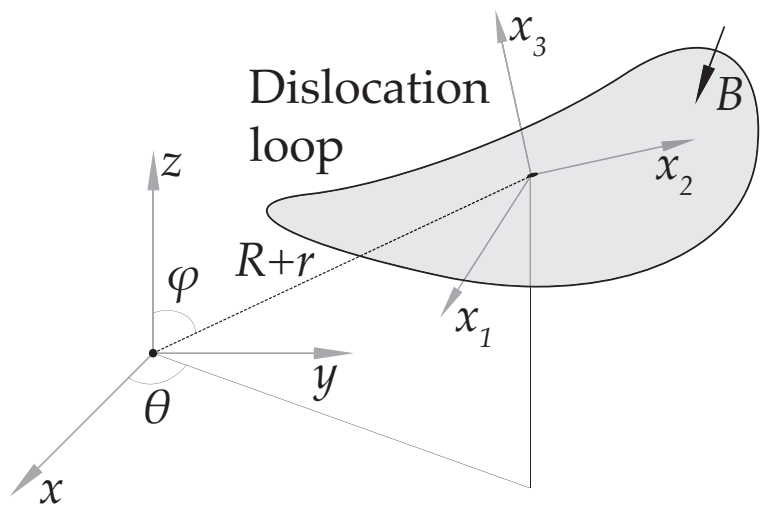

FIG. 4. Configuration of the three-dimensional system

\subsection{Stress field due to a stochastic cloud of dislocation loops}

We are now set to describe the corresponding stress tensor in spherical polar coordinates about the point we wish to evaluate the stress field at the origin. The dipolar expansion of the dislocation loops enables us to subsume the loop into a single point (the centre of area of the enclosed slipped surface), the long range field of which is an accurate approximation of the actual loop's elastic fields. As in the 2D case, we distinguish between the position of the centre of mass, and the inherent angular characteristics associated with the loop (i.e., the slip system and the orientation of the Burgers vector). Accordingly, we set a spherical polar coordinate system $(r, \theta, \phi)$ at a point of interest, which without loss of generality we choose to be the origin ; the position of the centre of area of the $n^{\text {th }}$ loop shall then be given by $\left(R+r_{n}, \theta_{n}, \phi_{n}\right)$ with $r_{n} \in R^{+}, \theta_{n} \in[0,2 \pi], \phi_{n}=[0, \pi]$.

These three random variables determine the spatial point process, associated to which we establish a number of marks. As depicted in fig.4, we have considered a secondary coordinate system with origin at the centre of area of the loop, $\left(x_{1}, x_{2}, x_{3}\right)$. Given that we are interested in the stress field at the origin of the global coordinate system $(x, y, z)$, we ought to rotate and translate the local coordinate system $\left(x_{1}, x_{2}, x_{3}\right)$. We employ a ZXZ Euler angle convention to that end, defining the $(\alpha, \beta, \gamma)$ rotation angles; these three Euler angles will serve as marks, and are defined by the crystallographic orientation of the slip system.

We restrict this study to dislocations lying on the primary slip system of an FCC or BCC crystal (albeit a similar one could be extended to HCP crystals). For simplicity, we set the $x y$ plane on the (0 10) plane; its normal lies on the $z=\left[\begin{array}{lll}0 & 1 & 0\end{array}\right]$ direction, and the global origin's direction along $x=\left[\begin{array}{lll}1 & 0 & 1\end{array}\right]$; accordingly, we set $y=\left[\begin{array}{ll}1 & 0\end{array}\right]$. When expressed in spherical coordinates, the $x$ axis acts as the origin of $\theta$, and the $z$ axis as the origin of $\phi$. The slip systems for FCC lie on the $\{111\}$ family of planes, and for BCC along the $\{110\}$; the slip directions are set along the $\langle 110\rangle$ for FCC crystals, and $\langle 111\rangle$ for BCC crystals [8]. The marks resulting from the corresponding slip systems, i.e., the Euler angles $(\alpha, \beta, \gamma)$, are summarised in table II.

The resulting point process shall therefore consist of the spatial random variables $N_{r \theta \phi}=\left\{r_{n}, \theta_{n}, \phi_{n}\right\} \in$ $R^{+} \times[0,2 \pi] \times[0, \pi] \equiv \mathbb{F}$, and the following marks: $A_{l_{n}} \in \mathbb{R}^{+} \equiv \mathbb{M}_{\mathrm{A}}$, which characterises the area enclosed by the loop; and $\left(\alpha_{n}, \beta_{n}, \gamma_{n}\right) \in[0,2 \pi] \times[0,2 \pi] \times[0,2 \pi] \equiv \mathbb{M}_{\text {slip }}$. We note that due to the way we have defined the Euler angles, it is necessary to account for the signature of the Burgers vector, for otherwise the direction of the loop will always be the same for each slip system; this shall make the last mark, $s_{n}=\{-1,1\} \equiv \mathbb{M}_{s}$. Thus, we have defined a marked point process $N=\mathbb{F} \times \mathbb{M}$ for $\mathbb{M}=\mathbb{M}_{\mathrm{A}} \times \mathbb{M}_{\text {slip }} \times \mathbb{M}_{s}$, which induces the collective intensity measure $\Lambda(\mathbb{F} \times \mathbb{M})=\nu(\mathbb{F}) \times \mu_{s}\left(\mathbb{M}_{s}\right) \times \mu_{\text {slip }}\left(\mathbb{M}_{\text {slip }}\right) \times \mu_{\mathrm{A}}\left(\mathbb{M}_{\mathrm{A}}\right)$. In the following we describe the distribution of marks and spatial points.

As can be seen, either case results in 12 slip systems, each of which we assume has equal probability; we note that the selection of one slip system sets the values of all three $\alpha_{n}, \beta_{n}, \gamma_{n}$ Euler angles. Hence, we may define the collective variable $\vartheta_{n} \equiv\left\{\alpha_{n}, \beta_{n}, \gamma_{n}\right\}$, which takes one of 12 values, and therefore follows the following probability density function

$$
f_{\vartheta}(\vartheta)=\frac{1}{12} \sum_{i=a \ldots l} \delta\left(\vartheta-\vartheta_{i}\right)
$$


FCC crystals

BCC crystals

\begin{tabular}{|c|c|c|c|c|c|c|c|c|c|c|c|}
\hline Label & Slip plane & Slip direction & $\cos \alpha$ & $\cos \beta$ & $\overline{\cos \gamma}$ & Labe & Slip plane & Slip direction & $\cos \alpha$ & $\cos \beta$ & $\cos \gamma$ \\
\hline $\mathrm{a}$ & & {$\left[\begin{array}{lll}1 & 0 & 1\end{array}\right]$} & & & 1 & $\mathrm{a}$ & $(110)$ & {$\left[\begin{array}{lll}1 & \overline{1} & 1\end{array}\right]$} & $\sqrt{2}$ & $\sqrt{2}$ & $-\frac{\sqrt{3}}{3}$ \\
\hline $\mathrm{b}$ & $\left(\begin{array}{lll}1 & 1 & 1\end{array}\right)$ & {$\left[\begin{array}{lll}0 & \overline{1} & 1\end{array}\right]$} & 0 & $\frac{\sqrt{3}}{3}$ & $\frac{1}{2}$ & $\mathrm{~b}$ & & {$\left[\begin{array}{lll}1 & 1 & 1\end{array}\right]$} & - & $\frac{12}{2}$ & $-\frac{\sqrt{3}}{3}$ \\
\hline $\mathrm{c}$ & & {$\left[\begin{array}{lll}1 & 1 & 0\end{array}\right]$} & & & $\frac{1}{2}$ & $\mathrm{c}$ & (10 1) & {$\left[\begin{array}{lll}1 & 1 & 1\end{array}\right]$} & 0 & 0 & $-\frac{\sqrt{6}}{3}$ \\
\hline $\mathrm{d}$ & & {$\left[\begin{array}{lll}1 & 0 & 1\end{array}\right]$} & & & -1 & $\mathrm{~d}$ & & {$\left[\begin{array}{lll}1 & 1 & \overline{1}\end{array}\right]$} & & & $-\frac{\sqrt{3}}{3}$ \\
\hline e & 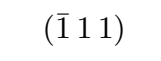 & {$\left[\begin{array}{lll}0 & \overline{1} & 1\end{array}\right]$} & 1 & $\frac{\sqrt{3}}{3}$ & $-\frac{1}{2}$ & $\mathrm{e}$ & $(1 \overline{1} 0)$ & {$\left[\begin{array}{lll}1 & 1 & 1\end{array}\right]$} & $\sqrt{2}$ & $\sqrt{2}$ & \\
\hline $\mathrm{f}$ & & {$\left[\begin{array}{lll}1 & 1 & 0\end{array}\right]$} & & & $-\frac{1}{2}$ & $\mathrm{f}$ & & {$\left[\begin{array}{lll}1 & 1 & \overline{1}\end{array}\right]$} & $-\frac{\overline{2}}{2}$ & $\overline{2}$ & $-\frac{\sqrt{3}}{3}$ \\
\hline $\mathrm{g}$ & & {$\left[\begin{array}{lll}1 & 0 & 1\end{array}\right]$} & & & 1 & $\mathrm{~g}$ & $(01$ & {$\left[\begin{array}{lll}1 & 1 & \overline{1}\end{array}\right]$} & $\sqrt{2}$ & $\sqrt{2}$ & $-\frac{\sqrt{3}}{3}$ \\
\hline $\mathrm{h}$ & $(1 \overline{1} 1)$ & {$\left[\begin{array}{lll}0 & 1 & 1\end{array}\right]$} & 0 & $-\frac{\sqrt{3}}{3}$ & $\frac{1}{2}$ & $\mathrm{~h}$ & $(011)$ & {$\left[\begin{array}{lll}1 & \overline{1} & 1\end{array}\right]$} & - & 2 & $-\frac{\sqrt{3}}{3}$ \\
\hline $\mathrm{Ii}$ & & {$\left[\begin{array}{lll}1 & 1 & 0\end{array}\right]$} & & & $-\frac{1}{2}$ & $\mathrm{i}$ & $(10 \overline{1})$ & {$\left[\begin{array}{lll}1 & 1 & 1\end{array}\right]$} & -1 & 0 & $\frac{\sqrt{6}}{3}$ \\
\hline $\mathrm{j}$ & & {$\left[\begin{array}{lll}1 & 0 & 1\end{array}\right]$} & & & -1 & $\mathrm{j}$ & $(101)$ & {$\left[\begin{array}{lll}1 & \overline{1} & 1\end{array}\right]$} & & & $\frac{\sqrt{6}}{3}$ \\
\hline $\mathrm{k}$ & $(\overline{1} \overline{1} 1)$ & {$\left[\begin{array}{lll}1 & 1 & 0\end{array}\right]$} & 1 & $-\frac{\sqrt{3}}{3}$ & $\frac{1}{2}$ & $\mathrm{k}$ & $(01 \overline{1})$ & {$\left[\begin{array}{lll}1 & 1 & 1\end{array}\right]$} & & $\sqrt{2}$ & $\frac{\sqrt{3}}{3}$ \\
\hline 1 & & {$\left[\begin{array}{lll}0 & 1 & 1\end{array}\right]$} & & & $-\frac{1}{2}$ & 1 & (0) & {$\left[\begin{array}{lll}1 & 1 & 1\end{array}\right]$} & & & $-\frac{\sqrt{3}}{3}$ \\
\hline
\end{tabular}

TABLE II. Euler angles for the 12 different slip systems of FCC (left) and BCC (right) crystals.

Equally, the signature is allocated with equal probability, so that

$$
f_{s}\left(s_{n}\right)=\frac{1}{2}\left(\delta\left(s_{n}-1\right)+\delta\left(s_{n}+1\right)\right)
$$

As in the $2 \mathrm{D}$ case, we must assume that the area enclosed by the loop will have an upper and a lower bound, $A_{\min }$ and $A_{\max }$. These ought to correspond to physical limitations: the size of the crystal and the minimum size required for producing a stable loop. The latter can be estimated by balancing the ideal lattice strength $\tau_{\text {ideal }} \approx \mu /(4 \pi)$ with self attracting forces experienced by the edge components of the loop (owing to these being larger than those experienced by the screw components); assuming that the loop is approximately circular with radius $\rho$, this sets

$$
\tau_{\text {ideal }} \equiv \frac{\mu}{4 \pi}=\frac{\mu B}{2 \pi(1-\nu)} \frac{1}{2 \rho} \Longrightarrow \rho=\frac{B}{1-\nu} \Longrightarrow A_{\min }=\pi \frac{B^{2}}{(1-\nu)^{2}} \approx 10 B^{2}
$$

In turn, the former $A_{\max }$ is defined as the typical grain size of the system under consideration, i.e., $A_{\max }=D$. As a first approach, we shall assume that the areas are uniformly distributed, so that

$$
f_{A}\left(A_{n}\right)=\frac{1}{A_{\max }-A_{\min }}
$$

The computation of the stress field components at the origin is elaborate enough that it merits some consideration. The dipolar field components given in table I are relative to the $\left(x_{1}, x_{2}, x_{3}\right)$ coordinate system, which is oriented relative to the global $(x, y, z)$ system as described with the Euler angles given in table II. We therefore seek to first rotate both the stress field components and the field variables in table I to align $\left(x_{1}, x_{2}, x_{3}\right)$, by rotating both coordinates and stress field components in table I first via the rotation matrix $L$

$$
L=\left(\begin{array}{ccc}
c_{\alpha} c_{\beta} c_{\gamma}-s_{\alpha} s_{\gamma} & -c_{\gamma} s_{\alpha}-c_{\alpha} c_{\beta} s_{\gamma} & c_{\alpha} s_{\beta} \\
c_{\beta} c_{\gamma} s_{\alpha}+c_{\alpha} s_{\gamma} & c_{\alpha} c_{\gamma}-c_{\beta} s_{\alpha} s_{\gamma} & s_{\alpha} s_{\beta} \\
-c_{\gamma} s_{\beta} & s_{\beta} s_{\gamma} & c_{\beta}
\end{array}\right)
$$

and then translating it back to the origin of the $(x, y, z)$ system with the linear transformation $x \mapsto x-x_{0}$, $y \mapsto y-y_{0}, z \mapsto z-z_{0}$, where $x_{0}, y_{0}, z_{0}$ describe the cartesian position of the centre of area of the loop relative to the origin. The resulting fields are then expressed in spherical polar coordinates $(\rho, \vartheta, \psi)$, and are then evaluated for $\rho=0, \vartheta=0, \psi=0$. Finally, we set $x_{0}=(r+R) \cos \theta \sin \phi, y_{0}=(r+R) \sin \theta \sin \phi$ and $z_{0}=(r+R) \cos \phi$.

The resulting field expressions are collected on table III. As can be seen on table III, all stress field components may be expressed as

$$
\sigma_{i j}=\frac{K_{i j}}{(r+R)^{3}} s A_{l} \Sigma_{i j}(\alpha, \beta, \gamma, \theta, \phi)
$$


where $K_{i j}$ is a constant dependent on the elastic constants, and $\Sigma_{i j}$ an angular term dependent on the marks $\alpha, \beta, \gamma$ and on the spatial angles $\theta, \phi$.

Thus, associated with the point process defined we have described above, we obtain the following fields

$$
\sigma_{i j}=K_{i j} \sum_{n=0}^{\infty} s_{n} A_{l_{n}} B_{n} \frac{\Sigma_{i j}\left(\alpha_{n}, \beta_{n}, \gamma_{n} ; \theta_{n}, \phi_{n}\right)}{\left(R+r_{n}\right)^{3}}=K_{i j} \sum_{\boldsymbol{x} \in N} f_{i j}(\boldsymbol{x})
$$

We may then proceed as in the 2D, and invoking Campbell's theorem compute the mean and the variance of the stress field components exerted by other dislocation loops over the origin. The averages are found to be

$$
\bar{\sigma}_{i j}=0
$$

whereas the variance, for the case of uniformly distributed loop areas, is of the form

$$
\operatorname{Var}\left[\sigma_{i j}\right]=\frac{B^{2} \mu^{2} \bar{\rho}_{\text {loop }}\left(A_{l_{\max }}^{2}+A_{l_{\max }} A_{l_{\min }}+A_{l_{\min }}^{2}\right)}{R^{4}(\lambda+2 \mu)^{2}} \varsigma_{i j}\left(\alpha_{1}, \ldots, \alpha_{12}, \beta_{1}, \ldots, \beta_{12}, \gamma_{1}, \ldots, \gamma_{12}\right)
$$

where $\bar{\rho}_{\text {loop }}=\bar{\phi} \bar{r} \bar{\theta}$ is the average number of loops per unit volume, which in turn is directly proportional to the dislocation density via $\bar{\rho}_{\text {loop }}=\bar{\rho}_{\text {dis }} / \bar{L}_{\text {loop}}$, with $\bar{L}_{\text {loop }}$ the mean length of a loop; and where $\varsigma_{i j}$ is an angular function that is directly proportional to the square of the elastic constants, and includes the slip system angles, and where as in the $2 \mathrm{D}$ case. Due to its size, it will not be reproduced here. We note however that if $\sqrt{A_{l}} \propto O(l)$, then the variance of the three dimensional case is considerably smaller than that of the two dimensional case. This shows that three dimensional dislocation structures attenuate one another considerably more than two dimensional ones. Furthermore, since the sign mark also premultiplies the higher order multipolar terms (e.g., the octopolar term given in section 44.1), the latter's contributions to the average stress field also vanish irrespective of the internal form of the multipolar field terms, and we may therefore conclude that plastic neutrality is maintained for 3D dislocation loops.

As in the $2 \mathrm{D}$ case, the same procedure may be extended for the case when the $A_{l}$ follows a log-normal distribution, which yields $\bar{\sigma}_{i j}=0$ and variances of the form:

$$
\operatorname{Var}\left[\sigma_{i j}\right]=\frac{B^{2} \mu^{2} \bar{\rho}_{\text {dis }} e^{2 m+2 s^{2}}}{\bar{L}_{\text {loop }} R^{4}(\lambda+2 \mu)^{2}} \varsigma_{i j}^{\log -\text { norm }}\left(\alpha_{1}, \ldots, \alpha_{12}, \beta_{1}, \ldots, \beta_{12}, \gamma_{1}, \ldots, \gamma_{12}\right)
$$

Thus, as in the 2D case, we observe that fully disordered clusters of dislocation loops entail plastic neutrality, and we must conclude that only insofar as we may induce a partial ordering of the cloud of dislocation loops we may observe a collective non-zero average effect of the latter over the material. Still, fully random collectives of dislocations are sometimes used to model forests of dislocations, which in turn are used to justify the motion of non-forest dislocations against fixed obstacles [86]. The plastic neutrality we have established in this analysis shows that the forest of dislocations will have no net effect, on average, over those moving dislocations, other than as obstacles that must be overcome through close range interactions.

\subsection{Partial orders in 3D}

As in the 2D cases, a partial order may be induced over the distribution of loops to better capture physically relevant situations where the cloud of dislocation loops has been generated by a directional load, or otherwise arises from the material's processing and previous damage. As will be seen in the following, the main conclusions of the $2 \mathrm{D}$ case still apply to the three dimensional case. The most immediate case is one where we enforce the signature of all loops to be the same, which in the 3D case is achieved by setting $s_{n}=1 \forall n$. In that event, we find that the average stress field is non zero, and takes the form

$$
\bar{\sigma}_{i j}=\frac{\pi B \bar{\rho}_{\mathrm{dis}} \bar{A} K_{i j}}{L_{\mathrm{loop}} R(\lambda+2 \mu)} \bar{\Sigma}_{i j}\left(\alpha_{1}, \ldots, \alpha_{12}, \beta_{1}, \ldots, \beta_{12}, \gamma_{1}, \ldots, \gamma_{12}\right)
$$

where $K_{i j}$ is a constant directly proportional to the elastic constants, $\bar{A}$ is the average slipped surface of a loop, and $\bar{\Sigma}_{i j}$ is a (lengthy) angular function also directly proportional to the elastic constants. The full average fields are reproduced in the Appendix due to their length.

The associated variances are of the form

$$
\operatorname{Var}\left[\sigma_{i j}\right]=\frac{B^{2} \bar{\rho}_{\text {dis }} L_{\text {loop }}\left(A_{l_{\max }}^{2}+A_{l_{\max }} A_{l_{\min }}+A_{l_{\min }}^{2}\right) K_{i j}^{\prime}}{R^{4}(\lambda+2 \mu)^{2}} \bar{\Sigma}_{i j}^{\prime}\left(\alpha_{1}, \ldots, \alpha_{12}, \beta_{1}, \ldots, \beta_{12}, \gamma_{1}, \ldots, \gamma_{12}\right)
$$




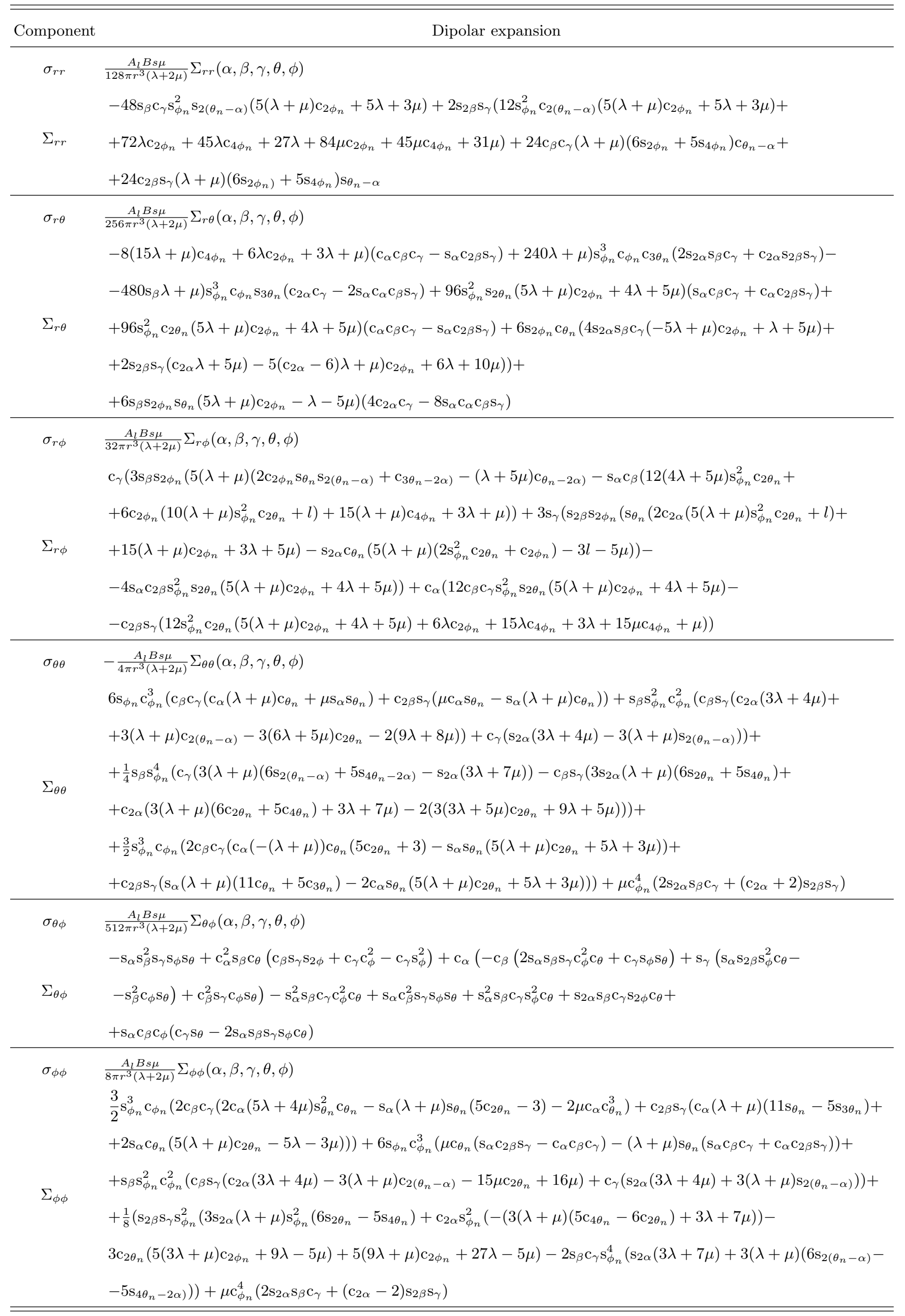

TABLE III. Force dipole expansion of the stress field of a planar dislocation loop in spherical polars, relative to the origin. Nota that here $c_{i} \equiv \cos (i), \mathrm{s}_{i} \equiv \sin (i)$ 
where $\bar{\Sigma}_{i j}^{\prime}$ is again a function of the slip plane angles and of the square of the elastic constants, and $K_{i j}^{\prime}$ a constant directly proportional to the square of the elastic constants as well.

Equation 49 is reminiscent of eqn.31, as in both cases the hardening is found to be directly proportional to the dislocation density and to a spatial lengthscale related to the average size of the individual dislocation cluster (dipole or loop, that is). The average distance between loops, $\|\bar{x}\|$, can be proven to remain, as per properties of the Poisson distribution, inversely proportional to the square root of the expectation of the intensity, which in turn we have defined as proportional to $\bar{\rho}_{\text {dis }} / L_{\text {loop }}$. Thus, $\|\bar{x}\| \propto 1 / \sqrt[3]{\bar{\rho}_{\text {dis }} / L_{\text {loop }}}$. Hence, if $\|\bar{x}\| \approx \sqrt[3]{\bar{A}^{2} / \bar{L}}$, then $\bar{\sigma}_{i j} \propto \sqrt{\bar{\rho}_{\text {dis }}}$, thereby recovering Taylor's equation.

Thus, it would seem that in 3D, obeying Taylor's equation binds the shape of the loop, measured by $\bar{A}$, to the length of its perimeter (i.e., of the dislocation line), $\bar{L}$, and these two variables to the average spacing between loops. This places a very particular expectation on the observed shape and spacing of the loops: the average distance between the loops must scale with $\sqrt[3]{A^{2} / \bar{L}}$. If the loops are such that their area-perimeter ratio is very large (for instance, very elongated), then they must be very far away from each other, and vice versa. In any other circumstance, Taylor's equation is not observed.

The spatial clustering of dislocations also renders similar conclusions. In particular, if we assume the intensity of the Poisson process to be the same as the one we considered a bivariate version of the $2 \mathrm{D}$ case described by eqn.28,

$$
\nu(x, y, z)=\frac{\bar{\nu}}{2 \pi|y||z| s_{y} s_{z} \sqrt{1-\rho^{2}}} e^{-\frac{q}{2}}
$$

with

$$
q=\frac{1}{1-\rho^{2}}\left[\frac{\ln |y|-m_{y}}{s_{y}}-\frac{\ln |z|-m_{z}}{s_{z}^{2}}\right]^{2}
$$

where $\rho$ is the correlation between $y$ and $z$ (which we may assume to be zero to begin with), and $m_{i}, s_{i}$ the lognormal means and standard deviations. Through this, we are in effect promoting the clustering of dislocation loops along a narrow band located at some distance defined by $m_{y}$ and $m_{z}$ from the origin; for simplicity hereafter we assume that $m_{y}=0$, we obtain that the mean stress at the origin is of the form

$$
\bar{\sigma}_{i j}=\frac{\pi B \bar{\rho}_{\mathrm{dis}} \bar{A} K_{i j}^{\prime} e^{-3 m_{z}+8 s_{z}^{4}}}{\bar{L}_{\mathrm{loop}} R(\lambda+2 \mu)} \bar{\varsigma}_{i j}\left(\alpha_{1}, \ldots, \alpha_{12}, \beta_{1}, \ldots, \beta_{12}, \gamma_{1}, \ldots, \gamma_{12}\right)
$$

where again $K_{i j}^{\prime}$ is a lengthy constant directly proportional to the elastic constants, and $\bar{\zeta}_{i j}$ a geometric function directly proportional to the elastic constants. The same scaling and conclusions apply in this case: Taylor's equation is recovered only if $\|\bar{x}\| \approx \sqrt[3]{\bar{A}^{2} / \bar{L}}$. We also observe an inverse relationship between $\bar{\sigma}_{i j}$ and the median distance between the two 'walls', which again implies that eqn.52 obeys the Hall-Petch relationship.

Thus, the crucial difference with the planar case is the area-perimeter requirement we have found for the dislocation loops in three dimensions. As in the $2 \mathrm{D}$ case, however, we still find two fundamental lengthscales governing the net stress state (the spacing between dislocations, and the area-perimeter ratio of the dislocation loops), and the Hall-Petch equation for wall-like structures.

a. Higher order contributions. If the variations in the stress field are of a similar lengthscale to the representative size of the loops, or if the dislocation cloud is dense, the dipolar approximation may not suffice in accurately capturing the short range stress fields between loops. In that event, the dipolar field formulation discussed here may have to be expanded to higher order multipolar terms such as those we have described in section 44.1 .

Given that the higher order contributions appear as summing terms in the fields expression, and because the expectation operator is linear, we may correct the average stress field at will by applying Campbell's theorem on the higher order multipolar terms contributions using the same marked process we have described above. We note that the $n^{\text {th }}$ order multipolar terms will depend on the $n-1$ moments of area of the dislocation loop, which as discussed in section 44.1 must be treated as additional marks in the point process.

As an example of the latter, in the following we focus on the significance the higher order multipolar terms have on the partial order where the signature of the loops is constant, and which leads to the Taylorlike equation. For instance, the octopolar field term given in section 44.1 (and collected in Appendix C) depends on the second moments of area, which were they to be treated as uniformly distributed marks (as we did with the loop's area), lead to the following correction term:

$$
\bar{\sigma}_{i j}^{(3)}=\frac{B \mu \bar{\rho}_{\text {dis }}}{\bar{L}_{\text {loop }}(\lambda+2 \mu) R^{3}} K_{i j}\left(\bar{J}_{p q}, \alpha, \beta, \gamma ; \mu, \lambda\right)
$$


where $\bar{J}_{p q}$ are the average second moments of area of the loop, and where $K_{i j}$ depends on the crystallography and the elastic constants. We do not reproduced it here due to its length; as an briefer example however, we will reproduce the $\bar{\sigma}_{r \theta}$ octopolar component for uniformly distributed moments of area in the 'e' crystallographic orientation of an FCC crystal in table II:

$$
\bar{\sigma}_{r \theta}^{(3)}=\frac{\pi \bar{\rho}_{\text {dis }} B \mu}{16,384 \sqrt{3} R^{3}(\lambda+2 \mu) \bar{L}_{\text {loop }}}\left(\bar{J}_{11}(534 \lambda+471 \mu)+\bar{J}_{22}(250 \lambda+301 \mu)\right)
$$

As can be seen, the octopolar terms will be directly proportional to the average moments of area. The octopolar term's average is added as a correction to the dipolar average term $\left(\bar{\sigma}_{i j}^{(1)}\right.$, given in eqn.49), so that

$$
\bar{\sigma}_{i j}=\bar{\sigma}_{i j}^{(1)}+\bar{\sigma}_{i j}^{(3)}
$$

provides the complete average stress field of interest, which now is affected by the dipolar and octopolar terms. Clearly, $\bar{\sigma}_{i j}^{(3)}$ dominates in the short range. It does not alter the $\bar{\rho}_{\text {dis }} / \bar{L}_{\text {loop }}$ proportionality found in eqn.49, so in principle it is possible to express $\bar{\sigma}_{i j} \propto \sqrt{\bar{\rho}_{\text {dis }}}$, thereby recovering Taylor's equation. However, the octopolar terms correct the $\sqrt{\bar{\rho}_{\text {dis }}}$ proportionality terms, which now will depend on some $\kappa^{(3)} \sqrt{\bar{\rho}_{\text {dis }}}$, where $\kappa=\bar{J}_{i j} / \bar{A}$ is the coefficient of variation of the loops' shape distribution, given that the second moments of area are the covariance of the latter. Thus, the loop's actual shape is seen to affect the average stress field in Taylor's equation, and it will become increasingly relevant if the latter is more disperse, since in that case for the same average distance between loop's centroids there being nearby dislocation lines becomes increasing likely.

Similarly, using higher order multipolar terms one could achieve additional correction terms dependent on the higher order moments of area. For the fourth order term, for instance, the average stress contribution may be shown to be proportional to the skewness-to-area ratio; the fifth to the kurtosis-to-area ratio, and so on. In general, we may say that

$$
\bar{\sigma}_{i j}^{(n)} \propto \frac{\bar{\rho}_{\text {dis }}}{\bar{L}_{\text {loop }} R^{n}} \bar{J}_{u_{1} \ldots u_{n-1}}
$$

where $\bar{J}_{u_{1} \ldots u_{n-1}}$ is the average $n-1$ moment of the loop.

The higher order multipolar field corrections to the average stress field are increasingly accurate over shorter distances. This is because the field contribution of the $n^{\text {th }}$ order multipolar field correction decays with $r^{n+1}$. The range over which each applies can be given as defined by the ratio between the prior and following multipolar moment of areas (see [50]). If we call $r_{n}$ to the minimum distance over which the $n^{\text {th }}$ order multipolar correction is valid, then accounting for the directionality of the moments of area,

$$
r_{n}=\min \left|\frac{\bar{J}_{u_{1} \ldots u_{n}}}{\bar{J}_{k_{1} \ldots k_{n-1}}}\right|
$$

This provides an estimate of the order of the multipolar correction needed to appropriately capture shorter range interactions at the desired lengthscale of the problem. The $2 n^{\text {th }}$ order statistical moments of the distribution of loop sizes are expected to be bounded by $n^{\text {th }}$ power of the variance [87], so $r_{n}$ is expected to converge to 0 .

What we therefore are able to show is that by taking the multipolar field expansion to increasingly higher orders, we can capture with increasing accuracy the short range interactions between dislocations, and that these reflect on the average stress fields as averages of the statistical moments of the distribution of loop shapes. This enables us to quantify, to an arbitrary accuracy, the latter's contributions to the collective stress state. In the limit, the multipolar fields converge to the actual fields of the loop, which would then be represented as complete, non-localised Burridge-Knopoff forces[50]. In such limit, one would recover the complete average stress state that could be computed from more sophisticated fibre process descriptions such as the ones considered in [48].

\section{DISCUSSION AND CONCLUSIONS}

This article provides a rigorous approach to computing the collective effect the collective effect that random populations of dislocations have on a crystalline material. In order to do so, we have employed the theory of stochastic point processes to introduce a disordered collection of dislocations in one, two, and three dimensions, and study the stress state they induce on a given material point. In each case, 
we have generated a spatially distributed stochastic population of dislocations according to some point process, and then evaluated the resulting stress fields by invoking Campbell's theorem.

In $1 \mathrm{D}$ and 2D, we have shown that the assumption that the populations of dislocations may consist of individual dislocations, which for instance commonly underpins the simulations of discrete dislocation plasticity (see [9]), may lead to statistical indeterminacy, i.e., to situations where either the mean stress state cannot be defined, or where even if the latter exists, the associated variance is infinite. This has been found to be because a single realisation of a distribution of randomly allocated individual dislocations is not guaranteed to conserve mass. We have then argued that, at least in $2 \mathrm{D}$, and the possibility of alternative constructions notwithstanding, mass conservation may be recovered if dislocations occur as dipoles of dislocations of same magnitude but opposite sign, separated by a given distance which we subsequently assumed to be a random variable as well.

In order to enforce mass conservation in $2 \mathrm{D}$, we have exploited the dipolar field expansion of a dipole of dislocations, which accurately describes the long-range fields of the dipole. In this way we have characterised the plastic neutrality of a disordered distribution of dislocation dipoles in 2D. The dipoles have been defined by their sign, crystallographic orientation relative to the base plane, and the separation distance between the dislocations forming the dipole; these three variables have been treated stochastically. The subsequent sum of their collective stress fields over the material point has been performed by stochastic integration, showing that the collective effect of the system of dislocations ought to be on average zero, i.e., that the material remains plastically neutral, but with standard deviation proportional to the square root of the dislocation density.

In 3D, we have considered a random population of closed dislocation loops of random shape. We have offer the dipolar expansion of a dislocation loop as a way to study the long range effect of the elastic fields of the loop. Further higher order multipolar field expansions have also been discussed, as a means of correcting the dipolar approximation in situations where the stress variation may be too large for the dipolar approximation to remain accurate. This has led to characterising the loop via its slipped (enclosed) area, the crystallographic orientation, and its signature. The latter were described as random variables, and the collective effect of a random population computed via stochastic integration based on Campbell's theorem for point processes. As in 2D, this enabled us to show the stress neutrality of a disordered population of dislocation loops (i.e., that their mean stress field over a specific point is zero), and that the associated standard deviation was proportional to the square root of dislocation density.

This shows that global plastic neutrality may be enforced is mass is conserved locally, i.e., if the dislocations do not occur individually but in loops or dipoles that ensure that locally mass is conserved as well as globally. The global elastic neutrality also shows that entirely disordered systems of dislocations will experience a strong, if not total, attenuation of their internal elastic fields.

At the same time, we have studied a number of circumstances where the cloud of dislocations was not fully disordered. In this study we have focused on aligning dislocations so that their collective long range effects would always be, locally, of the same sign, and in localising the cloud on narrow bands reminiscent of lamellar structures. Both situations have their physical basis, and are perhaps more realistic than an entirely disordered cloud of dislocations. In doing so, we have established the following scaling laws that generalise Taylor's own for the average plastic relaxation $\bar{\sigma}:(1)$ in $2 \mathrm{D}, \bar{\sigma} \propto \bar{l}_{\text {dipole }} \bar{\rho}_{\text {dis }}$; (2) in $3 \mathrm{D}$, $\bar{\sigma} \propto \bar{A}_{\text {loop }} \bar{L}_{\text {loop }} \bar{\rho}_{\text {dis }}$. Here $\bar{l}_{\text {dipole }}, \bar{A}_{\text {loop }}$, and $\bar{L}_{\text {loop }}$ are, respectively, the average length of a dipole, average slipped surface, and average length of a dislocation line, and where $\bar{\rho}_{\text {dis }}$ is the average dislocation density. Higher order multipolar corrections of the stress field average have shown the increasing role of the loops' shape in describing the near field, via the covariance, skewness, and higher order moments of the distribution of loop shapes. Furthermore, in the case of two opposed 'random' walls of dislocations, we determine that $\bar{\sigma} \propto 1 / \sqrt{D_{\text {grain }}}$, i.e., that the collective of dislocations in such cases obeys the Hall-Petch relation.

With regards to these scaling laws, we may therefore remark the following conclusions:

1. Fully disordered populations of dislocations, as the forest dislocations are sometimes assumed to be, are plastically neutral (i.e., do not contribute to the plastic hardening).

2. Locally clustered random populations of dislocations, and random populations of fully aligned dislocations, can exhibit a Taylor style equation.

3. We observe two fundamental length scales contributing to the plastic hardening of a material: the average spacing between dislocation loops, which is related to the dislocation density $\bar{\rho}_{\text {dis }}$, and the average size of those loops. If both length scales match, Taylor's equation is observed; if they do not, then the hardening becomes directly proportional to the dislocation density and to the area-perimeter ratio of the dislocation loop.

4. If the random clouds of dislocations are heavily localised in wall-like structures, the average stress 
scales with the inverse median distance between the walls, thereby obeying the Hall-Petch relationship.

Moreover, the mathematical procedure we have described focuses in evaluating the effect of an a priori unknown, random population of dislocations via methods of stochastic geometry, it could be extended to evaluating the local stress fields of experimentally observed populations of dislocations, and to verify whether the scaling conditions we have described are met.

A random population of dislocations is one in which the positions of individual dislocations are independent from one another, and allocated according to some point process of specific statistics. The constructive hypothesis of this study is that dislocations may be distributed in space according to a Poisson point process. This is tantamount to assuming that the location of one dislocation does not influence that of any other. A priori, this is a reasonable hypothesis if the material has been annealed, if the population arises from rare events such as radiation damage, or if the population of dislocations is superimposed to another that is already saturated and locked. It is also a reasonable assumption if one seeks to study long range effects, and the population of dislocations is heavily localised, since in that case the localisation effect will likely surpass the internal resolution of the structures.

However, there are instances where this hypothesis is no longer reasonable, particularly if the density of dislocations is large and the plasticity is heavily path dependent. Nevertheless, this does not mean that the methodology we have proposed here breaks down. As we have shown, the approximation of the loop's elastic fields via their dipolar moments may be extended to higher order terms, leading to arbitrarily more accurate expressions dependent on the higher order statistical moments of the distribution of loops, which as we have seen will be increasingly dominant in the near field; a similar approach could be employed in two dimensions if analogously, short range interactions had to be accounted with better accuracy. Furthermore, there exist alternative point processes the intensity of which models the internal interactions between points. An appropriate type of point process able to better capture such effect is the so-called Gibbs point process, where the distribution of points is affected by the neighbouring point's own attraction and repulsion [88]. Having said that, the modelling of such process is considerably challenging. The intensity measure of such point process depends on the system's total Hamiltonian, which in the case of an ensemble of points such as the one under consideration here is typically intractable owing to the large number of interacting particles involved, and is therefore not generally available. Future work will focus on extending the analysis presented here to stochastic processes involving interacting particles (using for instance a Gibbs process), and to specific studies of observable microstructures.

Thus, given a stochastic distribution of dislocations, this article has detailed how the global stress field (or, indeed, any other elastic field) can be computed. We have not homogenised the slip carried by dislocations along specific glide directions, nor have we focused on the population's evolution to external stimuli. In fact, we have shown and quantified that a given density of dislocations may induce radically different degrees of plastic relaxation on the material depending on the internal order of its constituent dislocations. Rather, we have concerned ourselves with deriving a methodology to compute the plastic state associated with any sort of dislocation populations, irrespective of their internal order or spatial localisation. In doing so, we have been able to show the most relevant salient macroscopic features of dislocation microstructures by studying the collective effect of random populations of dislocations. These collective effects have been studied under the theory of point processes and by relying on the multipolar expansions of dislocation clusters, and have been shown to lead to adequate scaling laws that generalise Taylor's equation, dislodging it from the strict square root dependency with the dislocation density for those situations where it might not hold true. These results are of immediate interest to the estimation of the stress state of large networks of dislocations. These may be experimentally observed, or obtained from large scale dislocation dynamics simulations. The main features of the dislocation network characterised in terms of the underlying point process (as is done for instance in geophysical applications[84]), and the stress fields evaluated as described in this article. They will also be useful in estimating the frictional stress experienced by a dislocation due to a cloud of background dislocations[8], and in generalising and randomising traditional regular patters of dislocations such as the Taylor lattice. We have introduced a simple, yet cost effective formalism to study such structures, and link them to experimentally measurable parameters. It has also allowed us to rigorously establish the statistical requirements that one should impose on random populations of dislocations in simulations of discrete dislocation dynamics, where such arrangements are often used as seeds.

\section{ACKNOWLEDGEMENTS}

The author is grateful to the Master and Fellows of Trinity College Cambridge for their munificent support under the author's Title A Fellowship. 


\section{APPENDICES}

\section{Appendix A. Multipolar expansions of edge dislocation dipoles}

The multipolar expansion of an edge dislocation dipole is attained from the multipolar expansion of a force distribution. Further details on this may be found in [50]. Here, it suffices to indicate that the elastic field of a body force distribution $f_{p}(\boldsymbol{x})$ acting on some infinite domain $\Omega$ is given by the representation theorem

$$
u_{i}(\boldsymbol{x})=\int_{\Omega^{\prime}} G_{i p}\left(\boldsymbol{x}-\boldsymbol{x}^{\prime}\right) f_{p}\left(\boldsymbol{x}^{\prime}\right) \mathrm{d} \Omega^{\prime}
$$

where Gip is the elastic Green's function. Expanding in Taylor series about $\boldsymbol{x}$, we find

$$
u_{i}(\boldsymbol{x})=\sum_{n=0}^{\infty} \frac{(-1)^{n}}{n !} \frac{\partial^{n} G_{i p}(\boldsymbol{x})}{\partial x_{k_{1}} \ldots \partial x_{k_{n}}} \gamma_{p k_{1} \ldots k_{n}}^{(n)}
$$

where $\gamma_{p k_{1} \ldots k_{n}}^{(n)}$ is the $n^{\text {th }}$ order multipolar moment, given by

$$
\gamma_{p k_{1} \ldots k_{n}}^{(n)}=\int_{\Omega^{\prime}}\left[x_{k_{1}}^{\prime} \cdot \ldots \cdot x_{k_{n}}^{\prime} f_{p}\left(\boldsymbol{x}^{\prime}\right)\right] \mathrm{d} \Omega^{\prime}
$$

A dipole of edge dislocations separated a distance $L$ from one another allows for the following BurridgeKnopoff force representation (see [89]), when the edge dislocation is given by $\left[u_{1}\right]=B\left[H\left(x_{1}\right)-H\left(x_{1}-L\right)\right]$,

$$
f_{1}=-B \mu\left[H\left(x_{1}\right)-H\left(x_{1}-L\right)\right] \delta^{\prime}\left(x_{2}\right), \quad f_{2}=-B \mu\left[\delta\left(x_{1}\right)-\delta\left(x_{1}-L\right)\right] \delta\left(x_{2}\right), \quad f_{3}=0
$$

where $H(\cdot)$ is the Heaviside function, $\delta(\cdot)$ the Dirac delta, $B$ the magnitude of the Burgers vector, $\mu$ the shear modulus. Accordingly, the first order, dipolar moment of the dipole of edge dislocations is

$$
\left.\gamma_{12}^{(1)}=\gamma_{21}^{(1}\right)=B \mu L
$$

Substituting this in the multipolar field expansion, we obtain $u_{i}(\boldsymbol{x})$ to dipolar order, and through differentiation, the stress fields. As is discussed in [50], these fields are extremely accurate in capturing the long range behaviour of the dipole. 


\section{Appendix B. Average dipolar field of a random distribution of dislocation loops}

The average dipolar fields at the origin for a random population of dislocation loops is given by:

$$
\begin{aligned}
& \bar{\sigma}_{r r}=\frac{\pi B \mu \bar{\rho}_{\text {dis }} 2 \bar{A}(27 \lambda+31 \mu)}{384 R(\lambda+2 \mu)}\left[\sin \left(\beta_{1}\right) \cos \left(\beta_{1}\right) \sin \left(\gamma_{1}\right)+\sin \left(\beta_{10}\right) \cos \left(\beta_{10}\right) \sin \left(\gamma_{10}\right)+\right. \\
& +\sin \left(\beta_{11}\right) \cos \left(\beta_{11}\right) \sin \left(\gamma_{11}\right)+\sin \left(\beta_{12}\right) \cos \left(\beta_{12}\right) \sin \left(\gamma_{12}\right)+\sin \left(\beta_{2}\right) \cos \left(\beta_{2}\right) \sin \left(\gamma_{3}\right)+\sin \left(\beta_{3}\right) \cos \left(\beta_{3}\right) \sin \left(\gamma_{3}\right)+ \\
& +\sin \left(\beta_{4}\right) \cos \left(\beta_{4}\right) \sin \left(\gamma_{4}\right)+\sin \left(\beta_{5}\right) \cos \left(\beta_{5}\right) \sin \left(\gamma_{4}\right)+\sin \left(\beta_{6}\right) \cos \left(\beta_{6}\right) \sin \left(\gamma_{6}\right)+\sin \left(\beta_{7}\right) \cos \left(\beta_{7}\right) \sin \left(\gamma_{7}\right)+ \\
& \left.+\sin \left(\beta_{8}\right) \cos \left(\beta_{8}\right) \sin \left(\gamma_{8}\right)+\sin \left(\beta_{9}\right) \cos \left(\beta_{9}\right) \sin \left(\gamma_{9}\right)\right] \\
& \bar{\sigma}_{r \theta}=\frac{\pi B \mu \bar{\rho}_{\mathrm{dis}} 2 \bar{A}(27 \lambda+31 \mu)}{384 R(\lambda+2 \mu)}\left[\sin \left(\beta_{1}\right) \cos \left(\beta_{1}\right) \sin \left(\gamma_{1}\right)+\sin \left(\beta_{10}\right) \cos \left(\beta_{10}\right) \sin \left(\gamma_{10}\right)+\right. \\
& +\sin \left(\beta_{11}\right) \cos \left(\beta_{11}\right) \sin \left(\gamma_{11}\right)+\sin \left(\beta_{12}\right) \cos \left(\beta_{12}\right) \sin \left(\gamma_{12}\right)+\sin \left(\beta_{2}\right) \cos \left(\beta_{2}\right) \sin \left(\gamma_{3}\right)+ \\
& +\sin \left(\beta_{3}\right) \cos \left(\beta_{3}\right) \sin \left(\gamma_{3}\right)+\sin \left(\beta_{4}\right) \cos \left(\beta_{4}\right) \sin \left(\gamma_{4}\right)+\sin \left(\beta_{5}\right) \cos \left(\beta_{5}\right) \sin \left(\gamma_{4}\right)+\sin \left(\beta_{6}\right) \cos \left(\beta_{6}\right) \sin \left(\gamma_{6}\right)+ \\
& \left.+\sin \left(\beta_{7}\right) \cos \left(\beta_{7}\right) \sin \left(\gamma_{7}\right)+\sin \left(\beta_{8}\right) \cos \left(\beta_{8}\right) \sin \left(\gamma_{8}\right)+\sin \left(\beta_{9}\right) \cos \left(\beta_{9}\right) \sin \left(\gamma_{9}\right)\right] \\
& \bar{\sigma}_{r \phi}=-\frac{\pi B \mu \bar{\rho}_{\mathrm{dis}} 2 \bar{A}(3 \lambda+\mu)}{384 R(\lambda+2 \mu)}\left[\sin \left(\alpha_{1}\right) \cos \left(\beta_{1}\right) \cos \left(\gamma_{1}\right)+\cos \left(\alpha_{1}\right) \cos \left(2 \beta_{1}\right) \sin \left(\gamma_{1}\right)+\right. \\
& +\sin \left(\alpha_{10}\right) \cos \left(\beta_{10}\right) \cos \left(\gamma_{10}\right)+\cos \left(\alpha_{10}\right) \cos \left(2 \beta_{10}\right) \sin \left(\gamma_{10}\right)+\sin \left(\alpha_{11}\right) \cos \left(\beta_{11}\right) \cos \left(\gamma_{11}\right)+ \\
& +\cos \left(\alpha_{11}\right) \cos \left(2 \beta_{11}\right) \sin \left(\gamma_{11}\right)+\sin \left(\alpha_{12}\right) \cos \left(\beta_{12}\right) \cos \left(\gamma_{12}\right)+\cos \left(\alpha_{12}\right) \cos \left(2 \beta_{12}\right) \sin \left(\gamma_{12}\right)+ \\
& +\sin \left(\alpha_{2}\right) \cos \left(\beta_{2}\right) \cos \left(\gamma_{2}\right)+\cos \left(\alpha_{2}\right) \cos \left(2 \beta_{2}\right) \sin \left(\gamma_{2}\right)+\sin \left(\alpha_{3}\right) \cos \left(\beta_{3}\right) \cos \left(\gamma_{3}\right)+ \\
& +\cos \left(\alpha_{3}\right) \cos \left(2 \beta_{3}\right) \sin \left(\gamma_{3}\right)+\sin \left(\alpha_{4}\right) \cos \left(\beta_{4}\right) \cos \left(\gamma_{4}\right)+\cos \left(\alpha_{4}\right) \cos \left(2 \beta_{4}\right) \sin \left(\gamma_{4}\right)+ \\
& +\sin \left(\alpha_{5}\right) \cos \left(\beta_{5}\right) \cos \left(\gamma_{4}\right)+\cos \left(\alpha_{5}\right) \cos \left(2 \beta_{5}\right) \sin \left(\gamma_{4}\right)+\sin \left(\alpha_{6}\right) \cos \left(\beta_{6}\right) \cos \left(\gamma_{6}\right)+\cos \left(\alpha_{6}\right) \cos \left(2 \beta_{6}\right) \sin \left(\gamma_{6}\right)+ \\
& +\sin \left(\alpha_{7}\right) \cos \left(\beta_{7}\right) \cos \left(\gamma_{7}\right)+\cos \left(\alpha_{7}\right) \cos \left(2 \beta_{7}\right) \sin \left(\gamma_{7}\right)+\sin \left(\alpha_{8}\right) \cos \left(\beta_{8}\right) \cos \left(\gamma_{8}\right)+\cos \left(\alpha_{8}\right) \cos \left(2 \beta_{8}\right) \sin \left(\gamma_{8}\right)+ \\
& \left.\left.+\sin \left(\alpha_{9}\right) \cos \left(\beta_{9}\right) \cos \left(\gamma_{9}\right)+\cos \left(\alpha_{9}\right) \cos \left(2 \beta_{9}\right) \sin \left(\gamma_{9}\right)\right)\right] \\
& \bar{\sigma}_{\theta \theta}=-\frac{2 \bar{A} B \mu \bar{\rho}_{\text {dis }} \pi}{3072(\lambda+2 \mu) R}\left[2(3 \lambda+19 \mu) \cos \left(\gamma_{1}\right) \sin \left(2 \alpha_{1}\right) \sin \left(\beta_{1}\right)+2(3 \lambda+19 \mu) \cos \left(\gamma_{10}\right) \sin \left(2 \alpha_{10}\right) \sin \left(\beta_{10}\right)+6 \lambda \cos \left(\gamma_{11}\right) \sin (20\right. \\
& \bar{\sigma}_{\theta \phi}=\frac{\pi B \mu \bar{\rho}_{\mathrm{dis}} 2 \bar{A}(3 \lambda+19 \mu)}{3072 R(\lambda+2 \mu)}\left[-\sin \left(2 \alpha_{1}\right) \sin \left(2 \beta_{1}\right) \sin \left(\gamma_{1}\right)+2 \cos \left(2 \alpha_{1}\right) \sin \left(\beta_{1}\right) \cos \left(\gamma_{1}\right)-\sin \left(2 \alpha_{10}\right) \sin \left(2 \beta_{10}\right) \sin \left(\gamma_{10}\right)+\right. \\
& +2 \cos \left(2 \alpha_{10}\right) \sin \left(\beta_{10}\right) \cos \left(\gamma_{10}\right)-\sin \left(2 \alpha_{11}\right) \sin \left(2 \beta_{11}\right) \sin \left(\gamma_{11}\right)+2 \cos \left(2 \alpha_{11}\right) \sin \left(\beta_{11}\right) \cos \left(\gamma_{11}\right)- \\
& -\sin \left(2 \alpha_{12}\right) \sin \left(2 \beta_{12}\right) \sin \left(\gamma_{12}\right)+2 \cos \left(2 \alpha_{12}\right) \sin \left(\beta_{12}\right) \cos \left(\gamma_{12}\right)-\sin \left(2 \alpha_{2}\right) \sin \left(2 \beta_{2}\right) \sin \left(\gamma_{2}\right)+ \\
& +2 \cos \left(2 \alpha_{2}\right) \sin \left(\beta_{2}\right) \cos \left(\gamma_{2}\right)-\sin \left(2 \alpha_{3}\right) \sin \left(2 \beta_{3}\right) \sin \left(\gamma_{3}\right)+2 \cos \left(2 \alpha_{3}\right) \sin \left(\beta_{3}\right) \cos \left(\gamma_{3}\right)- \\
& -\sin \left(2 \alpha_{4}\right) \sin \left(2 \beta_{4}\right) \sin \left(\gamma_{4}\right)+2 \cos \left(2 \alpha_{4}\right) \sin \left(\beta_{4}\right) \cos \left(\gamma_{4}\right)-\sin \left(2 \alpha_{5}\right) \sin \left(2 \beta_{5}\right) \sin \left(\gamma_{4}\right)+2 \cos \left(2 \alpha_{5}\right) \sin \left(\beta_{5}\right) \cos \left(\gamma_{4}\right)- \\
& -\sin \left(2 \alpha_{6}\right) \sin \left(2 \beta_{6}\right) \sin \left(\gamma_{6}\right)+2 \cos \left(2 \alpha_{6}\right) \sin \left(\beta_{6}\right) \cos \left(\gamma_{6}\right)-\sin \left(2 \alpha_{7}\right) \sin \left(2 \beta_{7}\right) \sin \left(\gamma_{7}\right)+2 \cos \left(2 \alpha_{7}\right) \sin \left(\beta_{7}\right) \cos \left(\gamma_{7}\right)- \\
& \left.-\sin \left(2 \alpha_{8}\right) \sin \left(2 \beta_{8}\right) \sin \left(\gamma_{8}\right)+2 \cos \left(2 \alpha_{8}\right) \sin \left(\beta_{8}\right) \cos \left(\gamma_{8}\right)-\sin \left(2 \alpha_{9}\right) \sin \left(2 \beta_{9}\right) \sin \left(\gamma_{9}\right)+2 \cos \left(2 \alpha_{9}\right) \sin \left(\beta_{9}\right) \cos \left(\gamma_{9}\right)\right] \\
& \sigma_{\phi \phi}=\frac{2 \bar{A} B \mu \bar{\rho}_{\mathrm{dis}} \pi}{3072(\lambda+2 \mu) R}\left[2(3 \lambda+19 \mu) \cos \left(\gamma_{1}\right) \sin \left(2 \alpha_{1}\right) \sin \left(\beta_{1}\right)+2(3 \lambda+19 \mu) \cos \left(\gamma_{10}\right) \sin \left(2 \alpha_{10}\right) \sin \left(\beta_{10}\right)+6 \lambda \cos \left(\gamma_{11}\right) \sin \left(2 \alpha_{1}\right.\right.
\end{aligned}
$$

\section{Appendix C. Octopolar field approximation}

The octopolar field components of a dislocation loop are given by:

$$
\begin{aligned}
\sigma_{11}^{3}= & \frac{1}{8 \pi\left(\lambda+2 \mu R^{11}\right.}\left[5 x _ { 1 } x _ { 3 } \left(\gamma_{1311}^{(3)}\left(\lambda\left(20 x_{1}^{4}-37 x_{1}^{2}\left(x_{2}^{2}+x_{3}^{2}\right)+6\left(x_{2}^{2}+x_{3}^{2}\right)^{2}\right)+3 \mu\left(8 x_{1}^{4}-12 x_{1}^{2}\left(x_{2}^{2}+x_{3}^{2}\right)+\left(x_{2}^{2}+x_{3}^{2}\right)^{2}\right)\right)\right.\right. \\
& -3 \gamma_{1322}^{(3)}\left(7 \lambda x_{1}^{2}\left(x_{1}^{2}-8 x_{2}^{2}+x_{3}^{2}\right)+\mu\left(8 x_{1}^{4}+x_{1}^{2}\left(9 x_{3}^{2}-61 x_{2}^{2}\right)-6 x_{2}^{4}-5 x_{2}^{2} x_{3}^{2}+x_{3}^{4}\right)\right)+12 \gamma_{3111}^{(3)} \lambda x_{1}^{4} \\
& -39 \gamma_{3111}^{(3)} \lambda x_{1}^{2} x_{2}^{2}-39 \gamma_{3111}^{(3)} \lambda x_{1}^{2} x_{3}^{2}+12 \gamma_{3111}^{(3)} \lambda x_{2}^{4}+24 \gamma_{3111}^{(3)} \lambda x_{2}^{2} x_{3}^{2}+12 \gamma_{3111}^{(3)} \lambda x_{3}^{4}+8 \gamma_{3111}^{(3)} \mu x_{1}^{4} \\
& -40 \gamma_{3111}^{(3)} \mu x_{1}^{2} x_{2}^{2}-40 \gamma_{3111}^{(3)} \mu x_{1}^{2} x_{3}^{2}+15 \gamma_{3111}^{(3)} \mu x_{2}^{4}+30 \gamma_{3111}^{(3)} \mu x_{2}^{2} x_{3}^{2}+15 \gamma_{3111}^{(3)} \mu x_{3}^{4}-15 \gamma_{3122}^{(3)} \lambda x_{1}^{4} \\
& +138 \gamma_{3122}^{(3)} \lambda x_{1}^{2} x_{2}^{2}-9 \gamma_{3122}^{(3)} \lambda x_{1}^{2} x_{3}^{2}-36 \gamma_{3122}^{(3)} \lambda x_{2}^{4}-30 \gamma_{3122}^{(3)} \lambda x_{2}^{2} x_{3}^{2}+6 \gamma_{3122}^{(3)} \lambda x_{3}^{4}-12 \gamma_{3122}^{(3)} \mu x_{1}^{4} \\
& \left.\left.+123 \gamma_{3122}^{(3)} \mu x_{1}^{2} x_{2}^{2}-3 \gamma_{3122}^{(3)} \mu x_{1}^{2} x_{3}^{2}-54 \gamma_{3122}^{(3)} \mu x_{2}^{4}-45 \gamma_{3122}^{(3)} \mu x_{2}^{2} x_{3}^{2}+9 \gamma_{3122}^{(3)} \mu x_{3}^{4}\right)\right]
\end{aligned}
$$




$$
\begin{aligned}
& \sigma_{22}^{3}=\frac{1}{8 \pi(\lambda+2 \mu) R^{11}}\left[5 x _ { 1 } x _ { 3 } \left(-\left(\gamma_{1311}^{(3)}+\gamma_{3111}^{(3)}\right)\left(21 \lambda x_{2}^{2}\left(-2 x_{1}^{2}+x_{2}^{2}+x_{3}^{2}\right)+\mu\left(4 x_{1}^{4}+x_{1}^{2}\left(x_{3}^{2}-41 x_{2}^{2}\right)+\right.\right.\right.\right. \\
& \left.\left.3\left(6 x_{2}^{4}+5 x_{2}^{2} x_{3}^{2}-x_{3}^{4}\right)\right)\right)+3 \gamma_{1322}^{(3)}\left(\lambda\left(2 x_{1}^{4}+x_{1}^{2}\left(4 x_{3}^{2}-31 x_{2}^{2}\right)+30 x_{2}^{4}-31 x_{2}^{2} x_{3}^{2}+2 x_{3}^{4}\right)\right. \\
& \left.+3 \mu\left(x_{1}^{4}+2 x_{1}^{2}\left(x_{3}^{2}-6 x_{2}^{2}\right)+8 x_{2}^{4}-12 x_{2}^{2} x_{3}^{2}+x_{3}^{4}\right)\right)+3 \gamma_{3122}^{(3)}\left(\lambda \left(2 x_{1}^{4}+x_{1}^{2}\left(4 x_{3}^{2}-31 x_{2}^{2}\right)+30 x_{2}^{4}\right.\right. \\
& \left.\left.\left.\left.-31 x_{2}^{2} x_{3}^{2}+2 x_{3}^{4}\right)+3 \mu\left(x_{1}^{4}+2 x_{1}^{2}\left(x_{3}^{2}-6 x_{2}^{2}\right)+8 x_{2}^{4}-12 x_{2}^{2} x_{3}^{2}+x_{3}^{4}\right)\right)\right)\right] \\
& \sigma_{33}^{3}=-\frac{1}{8 \pi(\lambda+2 \mu) R^{11}}\left[5 x _ { 1 } x _ { 3 } \left(\gamma_{1311}^{(3)} \lambda\left(8 x_{1}^{4}+2 x_{1}^{2}\left(x_{2}^{2}-20 x_{3}^{2}\right)-6 x_{2}^{4}+9 x_{2}^{2} x_{3}^{2}+15 x_{3}^{4}\right)\right.\right. \\
& +3 \gamma_{1311}^{(3)} \mu\left(4 x_{1}^{4}+x_{1}^{2}\left(x_{2}^{2}-13 x_{3}^{2}\right)-3 x_{2}^{4}+x_{2}^{2} x_{3}^{2}+4 x_{3}^{4}\right)+3 \gamma_{1322}^{(3)} \lambda\left(-2 x_{1}^{4}+x_{1}^{2}\left(10 x_{2}^{2}+3 x_{3}^{2}\right)\right. \\
& \left.+12 x_{2}^{4}-46 x_{2}^{2} x_{3}^{2}+5 x_{3}^{4}\right)+3 \gamma_{1322}^{(3)} \mu\left(-3 x_{1}^{4}+x_{1}^{2}\left(15 x_{2}^{2}+x_{3}^{2}\right)+18 x_{2}^{4}-41 x_{2}^{2} x_{3}^{2}+4 x_{3}^{4}\right) \\
& -42 \gamma_{3111}^{(3)} \lambda x_{1}^{2} x_{3}^{2}+21 \gamma_{3111}^{(3)} \lambda x_{2}^{2} x_{3}^{2}+21 \gamma_{3111}^{(3)} \lambda x_{3}^{4}-4 \gamma_{3111}^{(3)} \mu x_{1}^{4}-\gamma_{3111}^{(3)} \mu x_{1}^{2} x_{2}^{2}-43 \gamma_{3111}^{(3)} \mu x_{1}^{2} x_{3}^{2} \\
& +3 \gamma_{3111}^{(3)} \mu x_{2}^{4}+27 \gamma_{3111}^{(3)} \mu x_{2}^{2} x_{3}^{2}+24 \gamma_{3111}^{(3)} \mu x_{3}^{4}+21 \gamma_{3122}^{(3)} \lambda x_{1}^{2} x_{3}^{2}-168 \gamma_{3122}^{(3)} \lambda x_{2}^{2} x_{3}^{2}+21 \gamma_{3122}^{(3)} \lambda x_{3}^{4} \\
& \left.+3 \gamma_{3122}^{(3)} \mu x_{1}^{4}-15 \gamma_{3122}^{(3)} \mu x_{1}^{2} x_{2}^{2}+27 \gamma_{3122}^{(3)} \mu x_{1}^{2} x_{3}^{2}-18 \gamma_{3122}^{(3)} \mu x_{2}^{4}-183 \gamma_{3122}^{(3)} \mu x_{2}^{2} x_{3}^{2}+24 \gamma_{3122}^{(3)} \mu x_{3}^{4}\right)(81) \\
& \sigma_{12}^{3}=\frac{1}{8 \pi(\lambda+2 \mu) R^{11}}\left[5 x _ { 2 } x _ { 3 } \left(\gamma _ { 1 3 1 1 } ^ { ( 3 ) } \left(\lambda\left(30 x_{1}^{4}-31 x_{1}^{2}\left(x_{2}^{2}+x_{3}^{2}\right)+2\left(x_{2}^{2}+x_{3}^{2}\right)^{2}\right)+\mu\left(36 x_{1}^{4}-26 x_{1}^{2}\left(x_{2}^{2}+x_{3}^{2}\right)\right.\right.\right.\right. \\
& \left.\left.+\left(x_{2}^{2}+x_{3}^{2}\right)^{2}\right)\right)-3 \gamma_{1322}^{(3)}\left(21 \lambda x_{1}^{2}\left(x_{1}^{2}-2 x_{2}^{2}+x_{3}^{2}\right)+\mu\left(24 x_{1}^{4}+x_{1}^{2}\left(27 x_{3}^{2}-43 x_{2}^{2}\right)-4 x_{2}^{4}-x_{2}^{2} x_{3}^{2}+3 x_{3}^{4}\right)\right) \\
& +3(\lambda+\mu)\left(\gamma_{3111}^{(3)}\left(8 x_{1}^{4}-12 x_{1}^{2}\left(x_{2}^{2}+x_{3}^{2}\right)+\left(x_{2}^{2}+x_{3}^{2}\right)^{2}\right)-\gamma_{3122}^{(3)}\left(18 x_{1}^{4}+x_{1}^{2}\left(15 x_{3}^{2}-41 x_{2}^{2}\right)\right.\right. \\
& \left.\left.\left.\left.+4 x_{2}^{4}+x_{2}^{2} x_{3}^{2}-3 x_{3}^{4}\right)\right)\right)\right]
\end{aligned}
$$

$$
\begin{aligned}
& \sigma_{13}^{3}=\frac{1}{8 \pi(\lambda+2 \mu) R^{11}}\left[\gamma_{1311}^{(3)} \lambda\left(-12 x_{1}^{6}+3 x_{1}^{4}\left(3 x_{2}^{2}+53 x_{3}^{2}\right)+x_{1}^{2}\left(19 x_{2}^{4}-117 x_{2}^{2} x_{3}^{2}-136 x_{3}^{4}\right)-2\left(x_{2}^{2}-4 x_{3}^{2}\right)\left(x_{2}^{2}+x_{3}^{2}\right)^{2}\right)\right. \\
& +\gamma_{1311}^{(3)} \mu\left(-16 x_{1}^{6}+2 x_{1}^{4}\left(x_{2}^{2}+91 x_{3}^{2}\right)+x_{1}^{2}\left(17 x_{2}^{4}-96 x_{2}^{2} x_{3}^{2}-113 x_{3}^{4}\right)-\left(x_{2}^{2}-4 x_{3}^{2}\right)\left(x_{2}^{2}+x_{3}^{2}\right)^{2}\right) \\
& +15 \gamma_{1322}^{(3)} \lambda x_{1}^{2}\left(x_{1}^{4}-5 x_{1}^{2}\left(x_{2}^{2}+x_{3}^{2}\right)-6 x_{2}^{4}+51 x_{2}^{2} x_{3}^{2}-6 x_{3}^{4}\right)+3 \gamma_{1322}^{(3)} \mu\left(6 x_{1}^{6}-27 x_{1}^{4}\left(x_{2}^{2}+x_{3}^{2}\right)\right. \\
& \left.+x_{1}^{2}\left(-37 x_{2}^{4}+276 x_{2}^{2} x_{3}^{2}-37 x_{3}^{4}\right)-4 x_{2}^{6}+23 x_{2}^{4} x_{3}^{2}+23 x_{2}^{2} x_{3}^{4}-4 x_{3}^{6}\right)+120 \gamma_{3111}^{(3)} \lambda x_{1}^{4} x_{3}^{2}-180 \gamma_{3111}^{(3)} \lambda x_{1}^{2} x_{2}^{2} x_{3}^{2} \\
& -180 \gamma_{3111}^{(3)} \lambda x_{1}^{2} x_{3}^{4}+15 \gamma_{3111}^{(3)} \lambda x_{2}^{4} x_{3}^{2}+30 \gamma_{3111}^{(3)} \lambda x_{2}^{2} x_{3}^{4}+15 \gamma_{3111}^{(3)} \lambda x_{3}^{6}+8 \gamma_{3111}^{(3)} \mu x_{1}^{6}-16 \gamma_{3111}^{(3)} \mu x_{1}^{4} x_{2}^{2} \\
& +104 \gamma_{3111}^{(3)} \mu x_{1}^{4} x_{3}^{2}-21 \gamma_{3111}^{(3)} \mu x_{1}^{2} x_{2}^{4}-222 \gamma_{3111}^{(3)} \mu x_{1}^{2} x_{2}^{2} x_{3}^{2}-201 \gamma_{3111}^{(3)} \mu x_{1}^{2} x_{3}^{4}+3 \gamma_{3111}^{(3)} \mu x_{2}^{6}+24 \gamma_{3111}^{(3)} \mu x_{2}^{4} x_{3}^{2} \\
& +39 \gamma_{3111}^{(3)} \mu x_{2}^{2} x_{3}^{4}+18 \gamma_{3111}^{(3)} \mu x_{3}^{6}-90 \gamma_{3122}^{(3)} \lambda x_{1}^{4} x_{3}^{2}+765 \gamma_{3122}^{(3)} \lambda x_{1}^{2} x_{2}^{2} x_{3}^{2}-75 \gamma_{3122}^{(3)} \lambda x_{1}^{2} x_{3}^{4}-90 \gamma_{3122}^{(3)} \lambda x_{2}^{4} x_{3}^{2} \\
& -75 \gamma_{3122}^{(3)} \lambda x_{2}^{2} x_{3}^{4}+15 \gamma_{3122}^{(3)} \lambda x_{3}^{6}-12 \gamma_{3122}^{(3)} \mu x_{1}^{6}+69 \gamma_{3122}^{(3)} \mu x_{1}^{4} x_{2}^{2}-111 \gamma_{3122}^{(3)} \mu x_{1}^{4} x_{3}^{2}+69 \gamma_{3122}^{(3)} \mu x_{1}^{2} x_{2}^{4} \\
& \left.+828 \gamma_{3122}^{(3)} \mu x_{1}^{2} x_{2}^{2} x_{3}^{2}-81 \gamma_{3122}^{(3)} \mu x_{1}^{2} x_{3}^{4}-12 \gamma_{3122}^{(3)} \mu x_{2}^{6}-111 \gamma_{3122}^{(3)} \mu x_{2}^{4} x_{3}^{2}-81 \gamma_{3122}^{(3)} \mu x_{2}^{2} x_{3}^{4}+18 \gamma_{3122}^{(3)} \mu x_{3}^{6}\right] \\
& \sigma_{23}^{3}=-\frac{1}{8 \pi(\lambda+2 \mu) R^{11}}\left[5 x _ { 1 } x _ { 2 } \left(\gamma_{1311}^{(3)}(\lambda+\mu)\left(4 x_{1}^{4}+x_{1}^{2}\left(x_{2}^{2}-41 x_{3}^{2}\right)-3\left(x_{2}^{4}-5 x_{2}^{2} x_{3}^{2}-6 x_{3}^{4}\right)\right)\right.\right. \\
& -3 \gamma_{1322}^{(3)}(\lambda+\mu)\left(3 x_{1}^{4}-x_{1}^{2}\left(x_{2}^{2}+15 x_{3}^{2}\right)-4 x_{2}^{4}+41 x_{2}^{2} x_{3}^{2}-18 x_{3}^{4}\right)-42 \gamma_{3111}^{(3)} \lambda x_{1}^{2} x_{3}^{2}+21 \gamma_{3111}^{(3)} \lambda x_{2}^{2} x_{3}^{2} \\
& +21 \gamma_{3111}^{(3)} \lambda x_{3}^{4}-4 \gamma_{3111}^{(3)} \mu x_{1}^{4}-\gamma_{3111}^{(3)} \mu x_{1}^{2} x_{2}^{2}-43 \gamma_{3111}^{(3)} \mu x_{1}^{2} x_{3}^{2}+3 \gamma_{3111}^{(3)} \mu x_{2}^{4}+27 \gamma_{3111}^{(3)} \mu x_{2}^{2} x_{3}^{2} \\
& +24 \gamma_{3111}^{(3)} \mu x_{3}^{4}+63 \gamma_{3122}^{(3)} \lambda x_{1}^{2} x_{3}^{2}-126 \gamma_{3122}^{(3)} \lambda x_{2}^{2} x_{3}^{2}+63 \gamma_{3122}^{(3)} \lambda x_{3}^{4}+9 \gamma_{3122}^{(3)} \mu x_{1}^{4}-3 \gamma_{3122}^{(3)} \mu x_{1}^{2} x_{2}^{2}+81 \gamma_{3122}^{(3)} \mu x_{1}^{2} x_{3}^{2} \\
& \left.-12 \gamma_{3122}^{(3)} \mu x_{2}^{4}-129 \gamma_{3122}^{(3)} \mu x_{2}^{2} x_{3}^{2}+72 \gamma_{3122}^{(3)} \mu x_{3}^{4}\right)
\end{aligned}
$$

Here $R=\sqrt{x_{1}^{2}+x_{2}^{2}+x_{3}^{2}}$, and $\gamma_{3111}^{(3)}=B \mu J_{11}=\gamma_{1311}^{(3)}=\gamma_{1131}^{(3)}=\gamma_{1113}^{(3)}, \gamma_{1322}^{(3)}=\gamma_{1232}^{(3)}=\gamma_{1223}^{(3)}=\gamma_{3122}^{(3)}=$ $B \mu J_{22}$

[1] D. R. Nelson, M. Rubinstein, and F. Spaepen. Order in two-dimensional binary random arrays. Philosophical Magazine A, 46(1):105-126, 1982.

[2] W. Püschl, R. Frydman, and G. Schoeck. The strength of the dislocation forest for 30 and 60 dislocations. Physica status solidi (a), 74(1):211-216, 1982.

[3] E. Kröner. Benefits and shortcomings of the continuous theory of dislocations. International journal of solids and structures, 38(6-7):1115-1134, 2001. 
[4] P. B. Hirsch, A. Howie, R. B. Nicholson, D. W. Pashley, and M. J. Whelan. Electron Microscopy of Thin Crystals. Krieger, Boca Raton, FL, 1993.

[5] M. J. Whelan. Observations of Defects in Metals by Electron Microscopy. In P. B. Hirsch, editor, The Physics of Metals, volume 2, chapter 3, pages 98-151. Cambridge Univ. Press, 1975.

[6] D. F. Stein and J. R. Low Jr. Mobility of edge dislocations in silicon-iron crystals. J. Appl. Phys., 31(2):362369, 1960.

[7] A. S. Argon. Thermally-activated motion of dislocations through random localized obstacles. Philosophical Magazine, 25(5):1053-1072, 1972.

[8] J. P. Hirth and J. Lothe. Theory of Dislocations. John Wiley \& Sons, New York, 2nd edition, 1982.

[9] E. Van der Giessen and A. Needleman. Discrete dislocation plasticity: a simple planar model. Modelling Simul. Mater. Sci. Eng., 3(5):689-735, 1995.

[10] R. Madec, B. Devincre, and L. P. Kubin. From dislocation junctions to forest hardening. Physical review letters, 89(25):255508, 2002.

[11] Z. Q. Wang, I.J Beyerlein, and R. LeSar. Plastic anisotropy in fcc single crystals in high rate deformation. International Journal of Plasticity, 25(1):26-48, 2009.

[12] M. Zaiser, K. Bay, and P. Hähner. Fractal analysis of deformation-induced dislocation patterns. Acta Materialia, 47(8):2463-2476, 1999.

[13] D. M. Norfleet, D. M. Dimiduk, S. J. Polasik, M. D. Uchic, and M. J. Mills. Dislocation structures and their relationship to strength in deformed nickel microcrystals. Acta Materialia, 56(13):2988-3001, 2008.

[14] G. Saada. Sur l'interaction d'une dislocation fixe et d'une dislocation mobile dans son plan de glissement. Acta Metallurgica, 8(3):200-208, 1960.

[15] W. Püschl. Reactions between glide dislocations and forest dislocations in anisotropie bcc metals. physica status solidi (a), 90(1):181-189, 1985.

[16] B. Devincre, L. Kubin, and T. Hoc. Physical analyses of crystal plasticity by DD simulations. Scripta Materialia, 54(5):741-746, 2006.

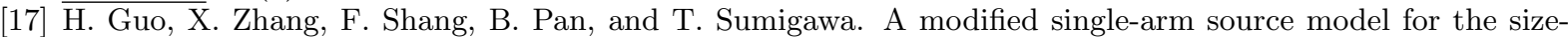
dependent strain-hardening behavior of metallic micropillars. International Journal of Mechanical Sciences, 133:438-448, 2017.

[18] A. El Azab. Statistical mechanics treatment of the evolution of dislocation distributions in single crystals. Physical Review B, 61(18):11956, 2000.

[19] J. Kratochvíl and R. Sedláček. Statistical foundation of continuum dislocation plasticity. Physical Review B, 77(13):134102, 2008.

[20] $\bar{S}$. Limkumnerd and E. Van der Giessen. Study of size effects in thin films by means of a crystal plasticity theory based on dift. Journal of the Mechanics and Physics of Solids, 56(11):3304-3314, 2008.

[21] B. L. Hansen, I. J. Beyerlein, C. A. Bronkhorst, E. K. Cerreta, and D. Dennis-Koller. A dislocation-based multi-rate single crystal plasticity model. International Journal of Plasticity, 44:129-146, 2013.

[22] J. Kratochvíl and M. Kružík. Statistically motivated model of mechanisms controlling evolution of deformation band substructure. International Journal of Plasticity, 81:196-208, 2016.

[23] M. G. Lee, H. Lim, B. L. Adams, J. P. Hirth, and R. H. Wagoner. A dislocation density-based single crystal constitutive equation. International Journal of Plasticity, 26(7):925-938, 2010.

[24] H. Wang, L. Capolungo, B. Clausen, and C. N. Tomé. A crystal plasticity model based on transition state theory. International Journal of Plasticity, 93:251-268, 2017.

[25] J. P. Sethna, M. K. Bierbaum, K. A. Dahmen, C. P. Goodrich, J. R. Greer, L. X. Hayden, J. P. KentDobias, E. D. Lee, D. B. Liarte, X. Ni, K. N. Quinn, A. Raju, D. Z. Rocklin, A. Shekhawat, and S. Zapperi. Deformation of crystals: Connections with statistical physics. Annual Review of Materials Research, 47, 2017.

[26] K. W. Schwarz. Discrete dislocation dynamics study of strained-layer relaxation. Physical review letters, 91(14):145503, 2003.

[27] D. S. Balint, V. S. Deshpande, A. Needleman, and E. Van der Giessen. Size effects in uniaxial deformation of single and polycrystals: a discrete dislocation plasticity analysis. Modelling Simul. Mater. Sci. Eng., 14:409-442, 2006

[28] M. A. Shehadeh, H. M. Zbib, and T. D. De la Rubia. Multiscale dislocation dynamics simulations of shock compression in copper single crystal. International Journal of Plasticity, 21(12):2369-2390, 2005.

[29] T. A. Parthasarathy, S. I. Rao, D. M. Dimiduk, M. D. Uchic, and D. R. Trinkle. Contribution to size effect of yield strength from the stochastics of dislocation source lengths in finite samples. Scripta Materialia, 56(4):313-316, 2007.

[30] C. Motz, D. Weygand, J. Senger, and P. Gumbsch. Initial dislocation structures in 3-d discrete dislocation dynamics and their influence on microscale plasticity. Acta Materialia, 57(6):1744-1754, 2009.

[31] B. Gurrutxaga-Lerma, D. S. Balint, D. Dini, D. E. Eakins, and A. P. Sutton. Dynamic Discrete Dislocation Plasticity, volume 47 of Advances in Applied Mechanics, chapter 2. Elsevier, 2014.

[32] Y. N. Cui, P. Lin, Z. L. Liu, and Z. Zhuang. Theoretical and numerical investigations of single arm dislocation source controlled plastic flow in fcc micropillars. International Journal of Plasticity, 55:279-292, 2014.

[33] B. Gurrutxaga-Lerma, D. S. Balint, D. Dini, D. E. Eakins, and A. P. Sutton. Attenuation of the dynamic yield point of shocked aluminum using elastodynamic simulations of dislocation dynamics. Phys. Rev. Lett., 114:174301, 2015. 
[34] E Tarleton, DS Balint, J Gong, and AJ Wilkinson. A discrete dislocation plasticity study of the microcantilever size effect. Acta Materialia, 88:271-282, 2015.

[35] E. Kröner. In M. F. Kanninen, W. F. Adler, A. B. Rosendfield, and R. I. Jaffee, editors, Inelastic Behavior of Solids, page 137. McGraw-Hill, New York, 1970.

[36] I. Groma. X-ray line broadening due to an inhomogeneous dislocation distribution. Physical Review B, 57(13):7535, 1998.

[37] I. Groma and B. Bakó. Probability distribution of internal stresses in parallel straight dislocation systems. Physical Review B, 58(6):2969, 1998.

[38] M. Zaiser, M.-C. Miguel, and I. Groma. Statistical dynamics of dislocation systems: The influence of dislocation-dislocation correlations. Physical Review B, 64(22):224102, 2001.

[39] S Yefimov, I Groma, and E Van der Giessen. A comparison of a statistical-mechanics based plasticity model with discrete dislocation plasticity calculations. Journal of the Mechanics and Physics of Solids, 52(2):279300, 2004.

[40] Sergey Yefimov, Erik Van der Giessen, and I Groma. Bending of a single crystal: discrete dislocation and nonlocal crystal plasticity simulations. Modelling and Simulation in Materials Science and Engineering, 12(6):1069, 2004.

[41] P. D. Ispánovity and I. Groma. The probability distribution of internal stresses in externally loaded $2 \mathrm{~d}$ dislocation systems. Journal of Statistical Mechanics: Theory and Experiment, 2008(12):P12009, 2008.

[42] M. Zaiser and I. Groma. Some limitations of dislocation walls as models for plast boundary layers. In AIP Conference Proceedings, volume 1389, pages 1540-1543. AIP, 2011.

[43] M. Zaiser. The energetics and interactions of random dislocation walls. Philosophical Magazine Letters, 93(7):387-394, 2013.

[44] M. Zaiser. Local density approximation for the energy functional of three-dimensional dislocation systems. Phys. Rev. B, 92(17):174120, 2015.

[45] Victor Berdichevsky. Energy of dislocation networks. International Journal of Engineering Science, 103:35-44, 2016.

[46] M. Zaiser and A. Seeger. Long-range internal stresses, dislocation patterning and work-hardening in crystal plasticity. In F. N. R. Nabarro and M. S. Duesbury, editors, Dislocations in Solids, volume 11, chapter 56, pages 1-100. North-Holland, Amsterdam, 2002.

[47] F. F. Csikor and I. Groma. Probability distribution of internal stress in relaxed dislocation systems. Physical Review B, 70(6):064106, 2004.

[48] A. El-Azab, J. Deng, and M. Tang. Statistical characterization of dislocation ensembles. Philosophical Magazine, 87(8-9):1201-1223, 2007.

[49] N. Gunkelmann, I. A. Alhafez, D. Steinberger, H. M. Urbassek, and S. Sandfeld. Nanoscratching of iron: A novel approach to characterize dislocation microstructures. Computational Materials Science, 135:181-188, 2017.

[50] B. Gurrutxaga-Lerma. Static and dynamic multipolar field expansions of defects in crystals: dislocations and cracks. Int. J. Engng. Sci., 128:165-186, 2018.

[51] G. I. Taylor. The mechanism of plastic deformation of crystals. Part I. Theoretical. Proc. Roy. Soc. A, 145:362-387, 1934.

[52] I. S. Gradshteyn and I. M. Ryzhik. Table of Integrals, Series and Products. Academic Press, New York, 7th edition, 2007.

[53] T. M. Apostol. Lerch's transcendent. In F. W. J. Olver, D. M. Lozier, R. F. Boisvert, and C. W. Clark, editors, NIST Handbook of Mathematical Functions. Cambridge Univ. Press, Cambridge, UK, 2010.

[54] L. M. Brown. Power laws in dislocation plasticity. Philosophical Magazine, 96(26):2696-2713, 2016.

[55] D. J. Daley and D. Vere Jones. An introduction to the theory of point processes. Volume II: general theory and structure. Springer, New York, 2007.

[56] J. R. Rice. Tensile crack tip fields in elastic-ideally plastic crystals. Mechanics of Materials, 6:317-335, 1987.

[57] M Wilkens. Das mittlere spannungsquadrat $\left\langle\sigma^{2}\right\rangle$ begrenzt regellos verteilter versetzungen in einem zylinderförmigen körper. Acta Metallurgica, 17(9):1155-1159, 1969.

[58] U. F. Kocks and R. O. Scattergood. Elastic interactions between dislocations in a finite body. Acta Metallurgica, 17(9):1161-1168, 1969.

[59] L. P. Kubin. Dislocations, Mesoscale Simulations and Plastic Flow, volume 5 of Oxford Series on Materials Modelling. Oxford Univ. Press, Oxford, UK, 2013.

[60] S. S. Shishvan and E. Van der Giessen. Distribution of dislocation source length and the size dependent yield strength in freestanding thin films. J. Mech. Phys. Solids, 58(5):678-685, 2010.

[61] A. A. Benzerga. An analysis of echaustion hardening in micron-scale plasticity. Int. J. Plasticity, 24:11281157,2008

[62] M. A. Meyers, F. Gregori, B. K. Kad, M. S. Schneider, D. H. Kalantar, B. A. Remington, G. Ravichandran, T. Boehly, and J. S. Wark. Laser-induced shock compression of monocrystalline copper: characterization and analysis. Acta Materialia, 51:1211-1228, 2003.

[63] D. D. Chambliss, R. J. Wilson, and S. Chiang. Nucleation of ordered ni island arrays on au (111) by surface-lattice dislocations. Physical review letters, 66(13):1721, 1991.

[64] W. Pantleon. On the statistical origin of disorientations in dislocation structures. Acta materialia, 46(2):451456, 1998. 
[65] D.J. Daley and D. Vere Jones. An Introduction to the Theory of Point Processes. Volume I: Elementary Theory and Methods. Springer, New York, 2nd edition, 2002.

[66] B. Gurrutxaga-Lerma, D. S. Balint, D. Dini, D. E. Eakins, and A. P. Sutton. The role of homogeneous nucleation in planar dynamic discrete dislocation plasticity. Journal of Applied Mechanics, 82(7):071008, 2015.

[67] C. S. Smith. Metallographic studies of metals after explosive shock. Trans. Met. Soc. AIME, 212:574-589, 1958.

[68] E. Hornbogen. Shock-induced dislocations. Acta Metallurgica, 10(10):978-980, 1962.

[69] M. A. Meyers. A mechanism for dislocation generation in shock-wave deformation. Scripta Metallurgica, 12:21-26, 1978.

[70] W. D. Nix and H. Gao. Indentation size effects in crystalline materials: a law for strain gradient plasticity. Journal of the Mechanics and Physics of Solids, 46(3):411-425, 1998.

[71] L. P. Kubin and A. Mortensen. Geometrically necessary dislocations and strain-gradient plasticity: a few critical issues. Scripta materialia, 48(2):119-125, 2003.

[72] HHM Cleveringa, E Van der Giessen, and A Needleman. A discrete dislocation analysis of bending. International Journal of Plasticity, 15(8):837-868, 1999.

[73] D. S. Balint, V. S. Deshpande, A. Needleman, and E. Van der Giessen. Discrete dislocation plasticity analysis of the grain size dependence of the flow strength of polycrystals. International Journal of Plasticity, 24(12):2149-2172, 2008.

[74] L. M. Brown and W. M. Stobbs. The work-hardening of copper-silica v. equilibrium plastic relaxation by secondary dislocations. Philosophical Magazine, 34(3):351-372, 1976.

[75] H. Mughrabi. Microstructural mechanisms of cyclic deformation, fatigue crack initiation and early crack growth. Phil. Trans. R. Soc. A, 373(2038):20140132, 2015.

[76] K. Hazeli, H. Askari, J. Cuadra, F. Streller, R. W. Carpick, H. M. Zbib, and A. Kontsos. Microstructuresensitive investigation of magnesium alloy fatigue. International Journal of Plasticity, 68:55-76, 2015.

[77] V. V. Bulatov and W. Cai. Computer simulations of dislocation. Oxford Univ. Press, Oxford, UK, 2006.

[78] D. Kuhlmann-Wilsdorf. Theory of plastic deformation:-properties of low energy dislocation structures. Materials Science and Engineering A, 113:1-41, 1989.

[79] UF Kocks and H Mecking. Physics and phenomenology of strain hardening: the fcc case. Progress in materials science, 48(3):171-273, 2003.

[80] L. E. Murr and D. Kuhlmann-Wilsdorf. Experimental and theoretical observations on the relationship between dislocation cell size, dislocation density, residual hardness, peak pressure and pulse duration in shock-loaded nickel. Acta Metallurgica, 26(5):847-857, 1978.

[81] J. D. Embury and J. P. Hirth. On dislocation storage and the mechanical response of fine scale microstructures. Acta metallurgica et materialia, 42(6):2051-2056, 1994.

[82] J. Jiang, T. B. Britton, and A. J. Wilkinson. The orientation and strain dependence of dislocation structure evolution in monotonically deformed polycrystalline copper. International Journal of Plasticity, 69:102-117, 2015.

[83] M. A. Meyers. Dynamic Behavior of Materials. John Wiley, Hoboken, NJ, 1994.

[84] S. N. Chiu, D. Stoyan, W. S. Kendall, and J. Mecke. Stochastic geometry and its applications. John Wiley \& Sons, Chichester, UK, 2013.

[85] T. Mura. Micromechics of Defects in Solids. Kluwer Academic Publishers, Amsterdam, 2nd edition, 1982.

[86] P. B. Hirsch. Work hardening. In P. B. Hirsch, editor, The Physics of Metals, volume 2, chapter 5, pages 189-246. Cambridge Univ. Press, 1975.

[87] J. Shohat. Inequalities for moments of frequency functions and for various statistical constants. Biometrika, 21(1-4):361-375, 1929.

[88] D. R. Cox and V. Isham. Point processes. Chapman and Hall, London, 1980.

[89] R. Burridge and L. Knopoff. Body force equivalents for seismic dislocations. Bulleting of the Seismological Society of America, 54(6):1875-1888, 1964. 